Citation: C. Pappalardo (2021). Una lettera di Paolo Bajnotti a Cristoforo Negri nel contesto dell'esplorazione del Mar Morto nel XIX secolo. Bollettino della Società Geografica Italiana serie 14, 4(1): 53-71. doi: 10.36253/bsgi-1357

Copyright: (C2021 C. Pappalardo. This is an open access, peer-reviewed article published by Firenze University Press (http://www.fupress.com/bsgi) and distributed under the terms of the Creative Commons Attribution License, which permits unrestricted use, distribution, and reproduction in any medium, provided the original author and source are credited.

Data Availability Statement: All relevant data are within the paper and its Supporting Information files.

Competing Interests: The Author(s) declare(s) no conflict of interest.

\section{Una lettera di Paolo Bajnotti a Cristoforo Negri nel contesto dell'esplorazione del Mar Morto nel XIX secolo}

\author{
A letter from Paolo Bajnotti to Cristoforo Negri in the context of \\ the exploration of the Dead Sea in the 19th Century
}

\author{
Carmelo Pappalardo \\ Dipartimento di Storia, Archeologia, Geografia, Arte e Spettacolo, Università degli Studi \\ di Firenze, Italia \\ E-mail: carmelo.pappalardo@gmail.com
}

\begin{abstract}
A letter in the archives of the Italian Geographical Society in which Paolo Bajnotti, an italian diplomat who had resided in Egypt, informs Cristoforo Negri about his trip to Palestine in 1869, before moving to Galaz for a new assignment, and about how he visited the Dead Sea thanks to the guidance of a Franciscan scholar who reported that he had personally observed that over the last ten years the level of the Dead Sea had dropped by about $95 \mathrm{~cm}$, provides the opportunity for a historical overview of the exploration of the Jordan Valley from Lake Tiberias to the Gulf of Aqaba during the 19 th century. What might seem to be a mere curiosity is in fact part of a very significant issue that was controversial among the geographers and cartographers of the time, as is clearly shown in Negri's speech at the meeting of the Società Geografica Italiana on March 13th 1870. The level of the Dead Sea and the level of the Sea of Galilee, the resulting difference in height that the River Jordan has to cover with a significant average gradient, the lack of a rise in the level of the Dead Sea despite the absence of an estuary were questions that in the mid-19th century were still waiting for an answer supported by scientific data and free of pious or pseudo-scientific overtones. A numerous series of expeditions - some improvised, others well organized - gave some answers to these issues, removing any doubt and establishing that the entire hydrologic basin was significantly below the level of the Mediterranean, that the Jordan did not have a strong slope because it was very meandering, and that the Dead Sea's level was regulated by the strong amount of evaporation that occurred throughout the year.
\end{abstract}

Keywords: Historical geography, Historical cartography, Geographical explorations, Dead Sea, Jordan River, Lake of Tiberias.

Riassunto. Una lettera conservata nell'archivio della Società Geografica Italiana nella quale Paolo Bajnotti, diplomatico torinese che aveva soggiornato in Egitto, racconta a Cristoforo Negri del suo viaggio in Palestina nel 1869, prima di trasferirsi a Galaz per un nuovo incarico, e di come avesse visitato il Mar Morto grazie alla guida di un dotto francescano che lo informa di come avesse avuto modo di appurare di persona che nel corso degli ultimi dieci anni il livello del Mar Morto si era abbassato di circa 95 $\mathrm{cm}$, diviene spunto per un excursus storico sull'esplorazione della valle del Giordano, 
dal Lago di Tiberiade fino al golfo di Aqaba, nel corso del XIX secolo. Infatti, quella che potrebbe sembrare una mera curiosità, si inserisce in una questione assai importante e dibattuta da geografi e cartografi di quel tempo, come ben evidenziato nel discorso del Negri all'adunanza della Società Geografica Italiana del 13 marzo 1870. Il livello del Mar Morto e quello del Lago di Galilea, il conseguente dislivello che il fiume Giordano deve coprire con una rilevante pendenza media, il mancato innalzamento del livello del Mar Morto a fronte della mancanza di un estuario erano problemi che alla metà dell' 800 attendevano ancora una risposta supportata da dati scientifici e scevra da commistioni religiose o pseudo-scientifiche. Una serie numerosa di spedizioni - alcune improvvisate, altre ben preparate - diedero delle risposte a queste domande, fugando ogni tipo di dubbio e stabilendo che tutto il bacino idrologico si trova abbondantemente sotto il livello del Mediterraneo, che il Giordano non ha una forte pendenza, perché assai sinuoso, e che il livello del Mar Morto era regolato dalla forte evaporazione che le sue acque subiscono nel corso dell'anno.

Parole chiave: Geografia storica, Cartografia storica, Esplorazioni geografiche, Mar Morto, fiume Giordano, lago di Tiberiade.

\section{Introduzione}

Conservata presso l'archivio della Società Geografica Italiana (U.A. 8, F.A., b. 3bis, fasc. 8$)^{1}$ vi è una breve lettera di Paolo Bajnotti ${ }^{2}$ indirizzata a Cristoforo Negri ${ }^{3}$, allora presidente della Società Geografica Italiana.

La missiva ${ }^{4}$, che consta di un unico foglio di carta ingiallita dal tempo, piegato a metà a formare le quattro facciate, su cui il testo è vergato ad inchiostro con scrit-

\footnotetext{
${ }^{1}$ Ringrazio la Società Geografica Italiana nelle persone della vicedirettrice Prof.ssa Margherita Azzari e della Responsabile della Biblioteca e degli Archivi dott.ssa Patrizia Pampana per avermi dato la possibilità di consultare, di fotografare, nonché di pubblicare la lettera.

${ }^{2}$ Il conte Paolo Bajnotti, nato a Torino il 26 aprile 1842 e morto a San Remo il 14 marzo 1919, fu un diplomatico del Regno d'Italia. Intraprese la carriera diplomatica sin da giovane, fino ad arrivare alla prestigiosa carica di ministro plenipotenziario d'Italia in America. Dalla lettera in studio sappiamo che nel 1869 venne trasferito dall'Egitto a Galatz, in Romania. [http://www.piemonteis.org/?p=2998]

${ }^{3}$ Cristoforo Negri (Milano, 13 giugno 1809 - Firenze, 18 febbraio 1896) fu un geografo, economista, diplomatico e politico italiano. Tra i fondatori della Società Geografica Italiana, ne fu il primo presidente dal 1867 al 1872 (Maggioli 2013).

${ }^{4}$ Segnatura archivistica: Società Geografica Italiana. Fondo Amministrativo / 1 Presidenza Cristoforo Negri / 8 corrispondenza 1870 - Ottobre-Dicembre / 9 Bajnotti Paolo/ Lettera 1 Paolo Bajnotti a Cristoforo Negri. Osservazioni riguardanti l'area della Giordania. [https:// patrimonio.archivio.senato.it/inventario/scheda/societa-geograficaitaliana-fondo-amministrativo/IT-AFS-028-002314/bajnotti-paolo] [https://patrimonio.archivio.senato.it/inventario/scheda/societa-geografica-italiana-fondo-amministrativo/IT-AFS-028-002315/paolo-bajnotticristoforo-negri]
}

tura sicura e leggibile, reca la data del 3 Ottobre 1869 e la località di Galatz ${ }^{5}$.

\section{Il testo}

\section{Galatz li 3 Ottobre 1869}

Ill.mo Signor Commendatore, / Prima di lasciare l'Egitto / per questa nuova mia residenza del Da-/nubio ho potuto procurarmi il piace-/re da lungo tempo desiderato di un / viaggio in Palestina ed, ospitato dai R. R. P. P. Francescani, / ebbi l'onore di conoscere uno di essi, / dotto archeologo e profondo studioso / di quelle contrade, e di fare in / carovana sotto la sua direzione la gita // di Gerico, del Giordano e del Mar Morto. I A riguardo del livello delle / acque di questo egli mi raccontò d'aver / osservato da dieci anni in qua un cos / tante ed ininterrotto abbassamento / che egli crede abbia raggiunto $i 95$ / centimetri per tale periodo di tempo. / Il fatto fu constatato dall'emersione / di certe ruine site nel lago vicino / alla foce del Giordano e che or fanno / dieci anni erano circa un metro sotto / il pelo delle acque. / Riservandomi a più minuti / ragguagli ed a dati precisi spero // non le sia discaro ch'io sottoponga / intanto anche cosi sommariamente / all'approvazione sapiente di V. / S. Ill.ma questo fenomeno che credo / sfuggito agli studi della spedizione / Americana di Lynch $^{6}$ e dell'Austriaca ${ }^{7}$ / e che parmi debba aumentare $i$ già / molti punti d'interesse che la / scienza trova in quella curiosa / regione che è il bacino del / Mar Morto e delle valli del / Cedron e del Giordano // inferiore. / Ho l'onore di profferirmele 8 / colla massima stima / Devotissimo / Paolo Bajnotti.

\section{Commento al testo}

Da quel che si evince dalle poche righe della lettera, Bajnotti informa il Negri che prima di lasciare il suo incarico diplomatico in Egitto, per un nuovo incarico a Galatz in Romania, ebbe l'opportunità di compiere un viaggio in Palestina, viaggio "da lungo tempo desiderato". Scarno di dettagli, il conte torinese ci fa sapere di essere stato ospite dei Padri Francescani della Custodia

\footnotetext{
${ }^{5}$ Nome tedesco della città di Galați sul Danubio nella neonata Romania, resasi indipendente dall'impero ottomano nel 1859, dopo la guerra di Crimea (1853-56).

${ }^{6}$ Infra.

${ }^{7}$ Con ogni probabilità fa riferimento alla spedizione diretta da J. Russegger che si recò sul Mar Morto per misurarne il livello nel corso dell'autunno del 1838 (infra).

${ }^{8}$ Espressione formale usata nella chiusura di una lettera, assai comune nel XIX secolo. Cfr. ad esempio A. Manzoni, Lettera 180, 22 febbraio 1823. http://www.alessandromanzoni.org/lettere/180
} 


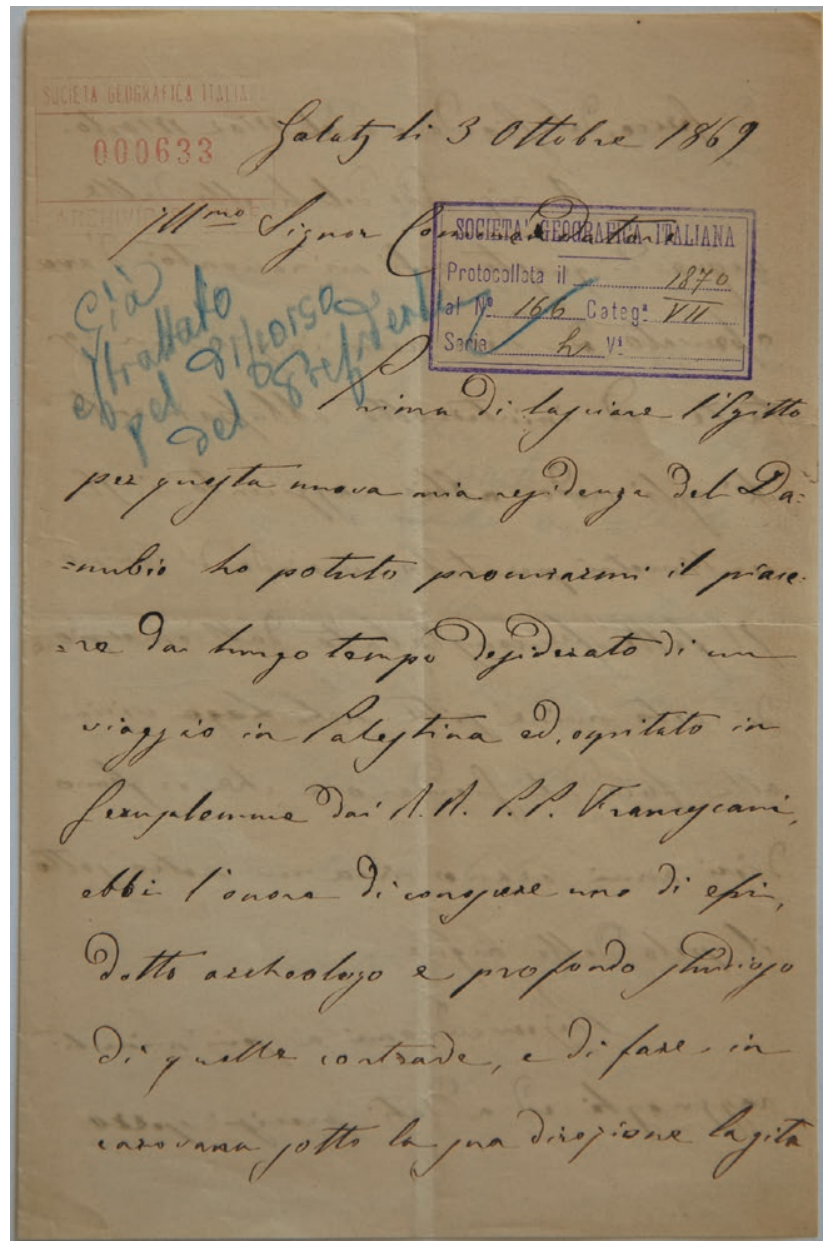

Figura 1. Il testo della lettera di Bajnotti a Negri, p. 1. Fonte: Società Geografica Italiana, Archivio storico (foto V. Bologna).

di Terra Santa a Gerusalemme9. Qui egli ha modo di conoscere un frate "dotto archeologo e profondo studioso di quelle contrade", che lo guida - "in carovana", cioè assieme ad altre persone - in una interessante escursione che lo porta a visitare Gerico, il fiume Giordano e il Mar Morto. Di tutto quel che vede lungo il viaggio e sul Mar Morto, l'unica cosa che ritiene interessante al punto di riferirlo espressamente al Presidente della Società Geografica Italiana, della quale egli era socio, è il dettaglio della diminuzione di $95 \mathrm{~cm}$ in dieci anni del livello del

\footnotetext{
${ }^{9}$ Di un edificio adibito dai Francescani all'ospitalità di pellegrini e personaggi illustri a Gerusalemme, la cosiddetta Casa Nova, si ha notizia già nel XVII secolo (Klimas 2015). Nei registri di Casa Nova, conservati nell'Archivio Storico della Custodia di Terra Santa (ASCTS), risulta che il Bajnotti vi ha soggiornato per 10 giorni, dal 2 al 12 di marzo del 1869. Di ciò si ha riscontro sia nel registro di minuta, che in quello ufficiale, dove però viene erroneamente trascritto il nome Carlo, anziché Paolo (ASCTS1874; ASCTS1869).
}

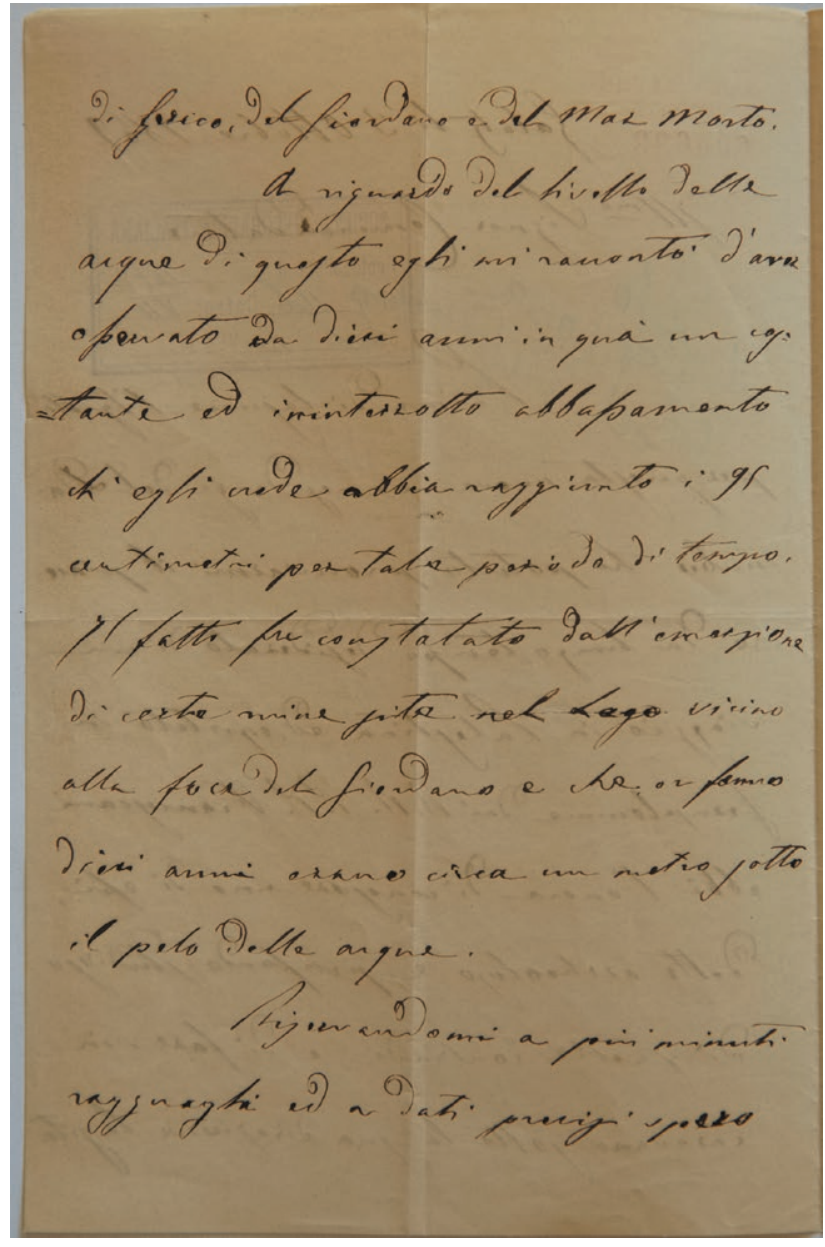

Figura 2. Il testo della lettera di Bajnotti a Negri, p. 2. Fonte: Società Geografica Italiana, Archivio storico (foto V. Bologna).

Mar Morto, così come gli era stato riferito dal frate che lo guidava.

\section{La reazione di Negri}

Tale notizia, evidentemente, non fu ritenuta di secondaria importanza, come evidenziato dal risalto che viene dato ad essa nel discorso tenuto dal Negri in occasione dell'adunanza della SGI del marzo 1870.

"Anche il socio Bajnotti ritornando da recente viaggio in Palestina, mi espose che il livello del Mar Morto (l'antico Frescobaldi piacevasi di chiamarlo Mare Maledetto ${ }^{10}$

${ }^{10}$ Il Negri fa qui riferimento all'appellativo dato al Mar Morto da Leonardo Frescobaldi, nobile fiorentino, nel resoconto che fece del suo viaggio in Terra Santa nel 1384-85. Viaggio di Lionardo di Niccolò Frescobaldi in Egitto e in Terra Santa (Frescobaldi 2012, 175). 


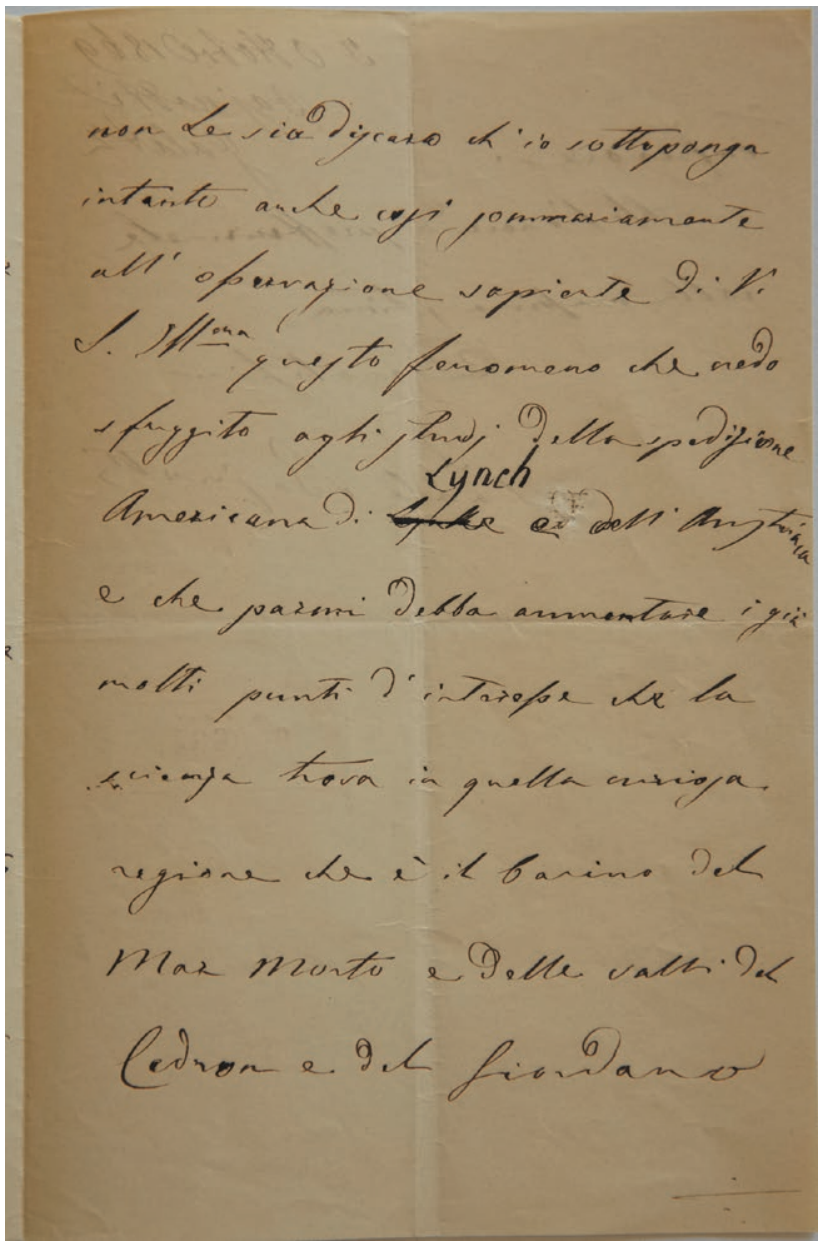

Figura 3. Il testo della lettera di Bajnotti a Negri, p. 3. Fonte: Società Geografica Italiana, Archivio storico (foto V. Bologna).

da dieci anni in poi è in costante abbassamento, e che le osservazioni fatte dai Padri di Terra Santa stabiliscono a 95 centimetri la variazione di livello avvenuta in questo periodo di tempo. Pregai il signor Bajnotti a procurarmi la serie delle osservazioni del fenomeno che ove fosse ben accertato, avrebbe molta importanza per la tanto discussa geologia di quella contrada. Su tale indicazione data dal socio Bajnotti, richiamai altresì l'attenzione particolare del distintissimo geografo Kiepert, che accompagnato da suo figlio Riccardo, giovane perspicace e di molta dottrina, partì per l'Egitto e la Siria ad esecuzione di incarichi scientifici meritatamente affidatigli dal Governo prussiano. Conobbi la sua partenza da lettera in data 28 febbraio, e ben volentieri raccomandai l'illustre Kiepert ai nostri soci che sono in Levante, ed ai molti miei amici nel corpo diplomatico e consolare italiano. Intanto sono lieto dell'ottima scelta fatta dal Governo di Prussia di un geografo eminente, che tanto pose di studi e fatiche nelle molte carte generali e speciali di Siria e di Egitto pubblicate da lui, conosce i problemi a sciogliere, e molti ne

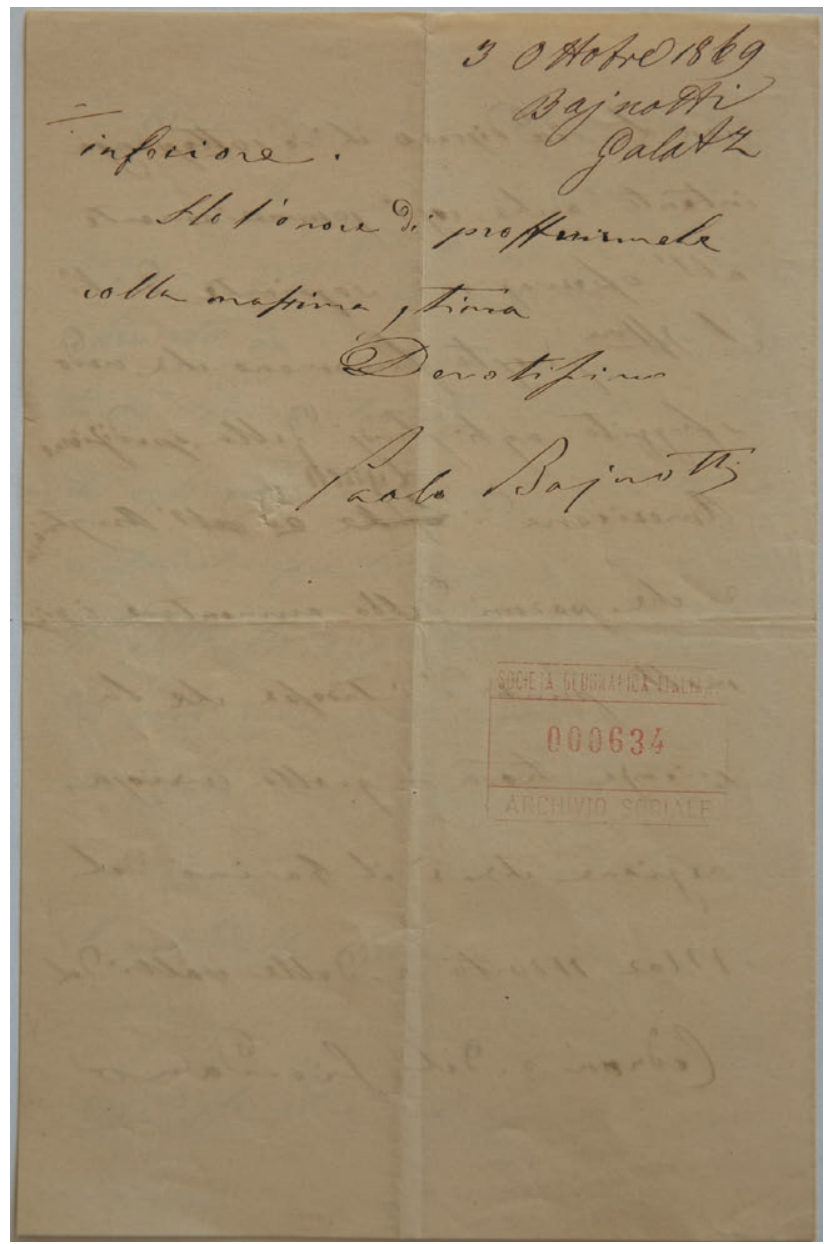

Figura 4. Il testo della lettera di Bajnotti a Negri, p. 4. Fonte: Società Geografica Italiana, Archivio storico (foto V. Bologna).

scioglierà, trovandosi ora a capo di bene allestita spedizione" (Negri 1870, 24-25).

Quella che potrebbe apparire come poco più di una curiosità, in realtà apporta un'importante novità e pone la nostra attenzione sulla questione dell'esplorazione della valle del Giordano e del Mar Morto che tanto aveva appassionato e impegnato, con risvolti a volte anche tragici, gli esploratori e geografi della metà dell' 800 .

\section{Il "dotto archeologo e profondo studioso di quelle contrade"}

Sull'identità del "dotto" frate che gli fa da guida si possono fare solo alcune supposizioni. Tra le attività dei Francescani di Terra Santa - presenti nel Vicino Oriente già a partire dalla prima metà del XIII secolo, ancora 
vivo San Francesco e ben prima della fondazione della Custodia di Terra Santa ${ }^{11}$ - vi è sempre stata quella di guidare ospiti e pellegrini a visitare quei territori e, in particolare, i luoghi nei quali, secondo antica tradizione, si sarebbero svolti gli eventi narrati nei Vangeli e negli altri libri della Bibbia. Non deve quindi stupire se, potendo contare su una conoscenza approfondita del territorio e su una tradizione plurisecolare, tra di loro ebbero a formarsi esimi studiosi.

\subsection{Francesco Cassini da Perinaldo}

Per quello che riguarda la metà del XIX secolo i nomi più importanti sono quelli di Francesco Cassini da Perinaldo che soggiornò in Terra Santa dal 1846 al 1854 e diede alle stampe, tra l'altro, un'interessante opera in tre volume dal titolo La Terra Santa descritta dal P. Francesco Cassini da Perinaldo, nella quale, parlando del Mar Morto, dice esplicitamente: "Ẽ questa la prima volta che vi parlo ex professo di un luogo, che non ho visitato, ma che ho solamente veduto di lontano" (Cassini da Perinaldo 1855, vol. 3, 124). Fa, inoltre, riferimento alla spedizione americana guidata da Lynch (infra):

“...quella commissione mandata tre anni fa dagli Stati Uniti d'America a scandagliare, ed a misurare il lago di Tiberiade, ed il lago Asfaltide: la quale commissione era composta di soggetti assai versati in queste materie, i quali avevano con sè una barca di ferro... Quei signori consumarono più di un mese nel fare le loro investigazioni, che pagarono ben care non solo di borsa, ma ancora di pelle" (Cassini da Perinaldo 1855, vol. 3, 132).

\subsection{Alessandro Bassi}

Un altro personaggio importante fu il francescano Alessandro Bassi che diede alle stampe nel 1857, anch'esso in tre volumi, il Pellegrinaggio storico e descrittivo di Terrasanta. Parlando del Mar Morto, ci informa che

“... è di circa 440 metri sotto il livello del mare, maggiore per conseguenza di quanti se ne conoscano su tutta la superficie del globo. Il Mar Morto è lungo circa ottantotto chilometri, largo ventidue. Spesso è ravvolto di densa caligine. Pei praticati scandagli risulta, che la sua maggiore profondità è di metri quattrocentotrenta... La salsedine delle acque è sei tanti quella del Mediterraneo" (Bassi 1857, vol.2, 215).

${ }^{11}$ Giuridicamente costituita nel 1342 da Clemente VI con la Bolla "Gratias agimus" (Piccirillo 2000, 146-147).
Egli descrive poi le sensazioni che prova immergendosi nell'acqua del mare, che, quindi, ha evidentemente visitato.

Entrambi si pongono il problema del mancato deflusso dell'acqua dal lago e riportano le varie spiegazioni, a volte pittoresche, del mancato aumento di livello dell'acqua, concordando, più o meno, sul fatto che la spiegazione più plausibile sia quella della forte evaporazione.

\subsection{Liévin de Hamme}

Ma la figura che con maggiore probabilità si cela dietro il laconico "dotto archeologo e profondo studioso" della nostra lettera è quella di Liévin de Hamme (de Looze 2016), una tra le più famose guide di pellegrini dell'ottocento. Nato l'11 agosto del 1822 ad Hamme in Belgio, Edouard Colleman all'età di 28 anni entra nell'Ordine dei Frati Minori a Gand, prendendo il nome di Fra Liévin. Nel 1856 si trasferisce a Parigi, dove presta servizio presso il locale Commissariato di Terra Santa. Ordinato sacerdote, viene mandato in Terra Santa, dove arriva il 6 gennaio del 1859 e dove morirà il 23 settembre 1898. Da subito si adoperò nella guida di pellegrini e visitatori illustri di varie nazionalità, dal momento che parlava olandese, francese, italiano e arabo, e comprendeva latino, tedesco e qualche parola di ebraico. Divenuto guida ufficiale di Terra Santa, approfondì gli studi di storia e geografia biblica e di archeologia. Si interessò anche dell'acquisto delle rovine di Tell Houm, la Cafarnao evangelica, e di quelle di Betfage, scoprendo qui la pietra affrescata d'epoca medievale che ricordava l'episodio evangelico dell'ingresso di Gesù a Gerusalemme (Luca 19, 29-40). Sembrerebbe quanto mai probabile che sia stato lui a guidare la "carovana" di cui faceva parte anche Paolo Bajnotti nel viaggio da Gerusalemme a Gerico, fiume Giordano e Mar Morto, e a Fra Liévin si dovrebbe quindi attribuire l'informazione sull'abbassamento di $95 \mathrm{~cm}$ in dieci anni del livello del Mar Morto (per coincidenza erano proprio dieci gli anni trascorsi da quando Fra Liévin era arrivato in Terra Santa).

Ma i dubbi tornano a palesarsi una volta lette le righe che il frate archeologo dedica al Mar Morto e alla regione di Gerico nella sua Guide-indicateur des sanctuaries et lieux historiques de la Terre-Sainte, opera in tre volumi data alle stampe a Gerusalemme prima nel 1869 e poi, in edizione rivista, nel 1887 :

“... on arrive au bord de la Mer Morte. ... On avance encore quelques pas et l'on descend de cheval sur un petit cap, vis-à-vis d'un îlot appelé Redjom Bahhr Louth 


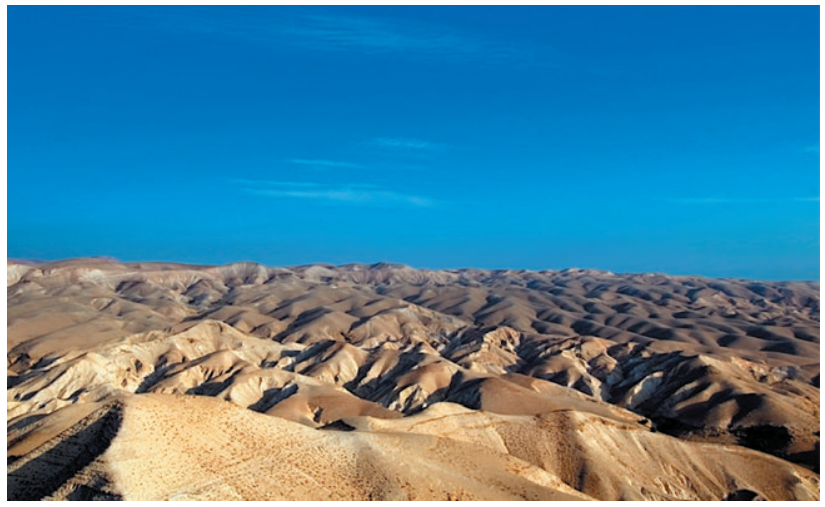

Figura 5. Il deserto di Giuda visto dalla strada da Gerusalemme a Gerico (foto dell'autore).

(le monceau de la mer de Loth) ${ }^{12}$... Cet îlot affecte grossièrement la forme circulaire. Il émerge au-dessus de la mer sur une étendue de 60 mèt. de diamètre, quelquefois moins encore ce qui dépend de la crue des eaux... (2) En 1860, j'ai pu me rendre deux fois à pied sec jusqu'à l'ilot. En 1861, mon cheval avait de l'eau jusqu'aux genoux ; en 1862, il en avait, dans certains endroits, jusqu'au ventre, et en 1863 les eaux avaient crû davantage. Depuis lors il m'a été impossible de m'y rendre si ce n'est en nageant. J'ai essayé souvent de prendre la mesure qui sépare l'ilot du rivage, mais la rapidité du courant et la force du vent faisaient tellement dériver la ficelle dont je me servais que la mesure prise ne pouvait être exacte, même approximativement. Une nouvelle tentative que j'essayais le 4 Janvier 1882, fut couronnée d'un plein succès. Vers onze heures du matin, par un temps tranquille, je me rendis au Redjom Bahhr Louth, emportant une forte corde dont j'avais lié une des extrémités à un tronc d'arbre placé au bord de la mer. Arrivé à l'ilot je me plaçai dans la direction du N. au S., puis je tendis bien la corde en tirant sur elle de toutes mes forces et je la coupai juste à l'endroit où elle touchait le mur de fondation de l'ancienne construction. La mesure obtenue fut de 243 mèt. 55 cent.: je ferai observer qu'à cette époque les grosses pluies hivernales n'étaient pas encore tombées, de sorte que la mer était basse" (De Hamme 1887, 281-282, n. 2).

Leggendo quanto scritto, si ha modo di apprezzare l'interesse del frate per il livello del mare che varia nel tempo, ma i dati che egli riferisce sembrano essere diametralmente opposti a quelli riportati da Bajnotti nella sua lettera. Cosa che rimane difficilmente spiegabile, se non ritenendo che sia stato un altro frate ad accompagnare Bajnotti e che le informazioni riferitegli a proposito del livello del mare fossero del tutto errate, oppure che sia stato lui a fraintendere quello che il frate gli

\footnotetext{
${ }^{12}$ Conder, Kitchener 1883, 177 e 220-221.
}

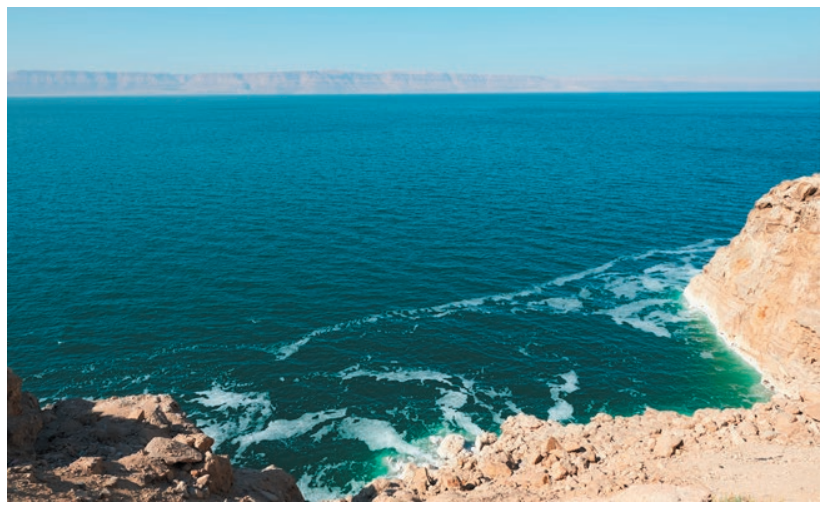

Figura 6. Il Mar Morto dalla sponda orientale all'altezza del Wadi Mujib/Arnun (foto dell'autore).

aveva detto. Indipendentemente da ciò, resta da notare il fatto che Bajnotti attiri l'attenzione su un dato assai importante, quello del livello del Mar Morto, la cui misurazione impegnò non poco le numerose spedizioni che si interessarono a quella arida regione nel volgere della metà del XIX secolo.

E Charles W. Wilson che, per conto del Palestine Exploration Fund, a partire dal 1865 si interessa del livello del Mar Morto e delle sue variazioni. Bisognerà tuttavia aspettare gli inizi del secolo scorso perché si cominci a monitorare in modo continuo e costante il livello del Mar Morto (infra). In tempi recenti alcuni studiosi si sono concentrati sulla ricostruzione dell'andamento del livello del Mar Morto nel corso del tempo, mostrando come, durante il XIX secolo, il livello del Mar Morto abbia avuto un andamento decrescente nel primo ventennio a toccare i $-400 \mathrm{~m}$, crescente nel secondo, poco fluttuante attorno ai $-395 \mathrm{~m}$ nei due decenni seguenti e costantemente in crescita nell'ultimo ventennio, fino a superare i $-390 \mathrm{~m}$ alla fine del secolo (Klein, Flohn 1987; si veda anche Gavrieli 2004; Kreiger 2016, 31-130). È quindi probabile che la notizia riportata dal Bajnotti non vada in contraddizione con quanto poi scritto da Fra Liévin nella sua Guide, ma piuttosto sia indizio dell'andamento fluttuante del livello del mare nel corso dei decenni centrali del secolo.

\section{L'esplorazione del Mar Morto nel corso del XIX secolo}

"Palestine's explorers of the new era differed from those who went to study other countries in one primary aspect, in the geopious ${ }^{13}$ influence that the subject of their studies

\footnotetext{
${ }^{13}$ Il termine geopiety o quello più generico geosophy è stato coniato dal
} 
had on their research. It was obvious that no one could ignore the uniqueness of Palestine, the 'Holy Land', with all its implications... The participants who undertook this scientific adventure did their best to perform their study according to the high scientific standards of their time, but they could not avoid the religious connection, and it strongly influenced the objects and topics they chose to investigate" (Goren 2016, 4).

\subsection{Ulrich Jasper Seetzen}

Il primo esploratore moderno a visitare la regione siro-palestinese fu il tedesco Seetzen (1767-1811) (Lichtenberger 2011). Egli attraversò la regione che comprende il lago di Tiberiade, la valle del Giordano e il Mar Morto tra il 1805 e il 1807. Le descrizioni riportate nei suoi diari, che egli annotò con quotidiana puntualità dal 1802 ad almeno tutto il 1809, sono in parte sommarie, concise e sobrie; in parte, invece, si dilungano in resoconti dettagliati e letterariamente elaborati: è il caso, per esempio, della perlustrazione delle rive del Mar Morto che compì più volte, senza però addentrarsi mai dentro le sue acque. In particolare affronterà il viaggio da Kerak a Gerusalemme attraverso la sponda meridionale del Mar Morto dal 2 al 10 aprile 1806; mentre dal 15 al 18 dicembre dello stesso anno esplora e descrive i territori della sponda occidentale del Mar Morto; dal 5 gennaio al 2 febbraio del 1807 esplora invece la sponda orientale e l'entroterra ${ }^{14}$.

\subsection{Johann Ludwig Burckhardt}

Lo svizzero Burckhardt ${ }^{15}$ (1784-1817) è forse il più famoso esploratore moderno del Vicino Oriente; a lui si deve, tra l'altro, la riscoperta moderna della leggendaria città nabatea di Petra. A lui si deve anche una dettagliata

geografo statunitense John K. Wright "per indicare quel complesso di relazioni e legami emotivi - attaccamento, reverenza, amore - che si stabiliscono tra l'uomo, o meglio un gruppo definito, ed il suo territorio". Lando 2012, 274, n. 44; cfr. anche Wright 1966, 7 e 251-253.

${ }^{14}$ I suoi diari vennero parzialmente pubblicati solo postumi nel 1854 in quattro volumi che raccoglievano i quaderni dal 1805 al 1809 (Seetzen 1854); in particolare per quanto riguarda la regione del Mar Morto cfr. Seetzen 1854, vol. 1, 426-431; vol. 2, 217-274, 293-385. Alcune sue note erano invece già state pubblicate in inglese nel 1810 a cura della Palestine Association of London (Seetzen 1810). Per la parte riguardante il suo viaggio in Palestina e dintorni si veda anche Seetzen 2002.

${ }^{15}$ Figlio di una famiglia di commercianti svizzeri, venne assunto dalla African Association di Londra e, dopo aver studiato arabo a Cambridge, si trasferì ad Aleppo, dove continuò a studiare l'arabo e il Corano facendosi chiamare Ibrahim bin Abdullah, nome che mantenne fino alla morte. Visse e viaggiò per tutto il Vicino Oriente, arrivando fino alla Mecca e Medina, fino in Nubia e scoprendo Petra e Abu Simbel. Morì di dissenteria al Cairo il 15 ottobre 1817. descrizione della regione che va dal Mar Morto al golfo di Aqaba nel volume Travels in Syria and the Holy Land pubblicato postumo nel 1822 a Londra (Burckhardt $1822)^{16}$. Nel viaggio da Damasco al Cairo attraverso l'altopiano transgiordanico, che compirà nell'estate del 1812, Burckhardt ha modo di osservare e descrivere il paesaggio attorno al Mar Morto ${ }^{17}$.

\subsection{Charles Irby e James Mangles}

Due ufficiali della Royal Navy inglese, Irby (17891845) e Mangles (1786-1867), nel 1817 intrapresero un viaggio nel vicino oriente che si protrasse per quattro anni. Non pubblicarono un vero resoconto del viaggio, ma vennero persuasi a dare alle stampe le lettere che avevano inviato ai loro familiari, dove il viaggio viene raccontato con dovizia di particolari (Irby, Mangles 1823). La parte riguardante l'itinerario dalle fonti del Giordano fino alla sponda meridionale del Mar Morto per risalire dalla sponda orientale fin su a Gerash, Tiberiade e concludersi ad Acri, si può trovare nelle lettere IV e V. Era il 6 maggio 1818 quando Irby e Mangles cominciarono il loro giro esplorativo attorno al Mar Morto con due arabi, scendendo da Hebron e risalendo sulla riva opposta in direzione di Kerak. Si accorsero che lo stretto del Lisan era guadabile durante alcuni periodi dell'anno, almeno al loro tempo, prima, cioè, che il livello del Mar Morto risalisse durante la seconda metà del XIX secolo (Irby, Mangles 1823, 352-355).

"We found, exclusive of the saline appearance left by the retiring of the waters, several large fragments of clear rock-salt lying on the ground, and on examining the hill, we found it composed partly of salt and partly of hardened sand. In many instances the salt was hanging from cliffs in clear perpendicular points like icicles, and we observed numerous strata of that material of considerable thickness, having very little sand mixed with it" (Irby, Mangles 1823, 352-355).

L'esplorazione del Mar Morto e la navigazione del Giordano partendo dal Lago di Galilea è, tuttavia, legata in particolare a tre esploratori della prima metà del XIX secolo: Christopher Costigan, Thomas H. Molineux e William F. Lynch. Tutt'e tre tentarono di navigare par-

\footnotetext{
16 "The principal geographical discoveries of our traveller, are the nature of the country between the Dead Sea and the gulf of AElana, now Akaba..." (Burckhardt, 1822, p. v).

${ }^{17}$ Tra le altre cose, a proposito del livello del Mar Morto egli annota quanto segue: "Concerning the lake itself, I was informed that no visible increase of its waters takes place in winter time, as the greater part of the torrents which descend from the eastern mountains do not reach the lake, but are lost in the sandy plain" (Burckhardt 1822, 394).
} 


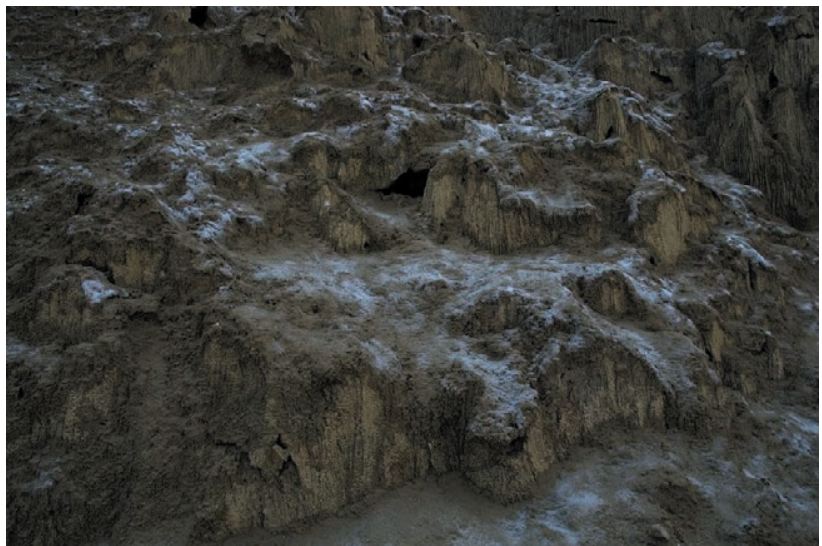

Figura 7. Le formazioni saline dei monti di Sedom come descritte da Irby e Mangles (foto dell'autore).

tendo dal Lago di Tiberiade attraverso il fiume Giordano per arrivare al Mar Morto (Masterman 1905; Masterman 1911). I primi due tentativi finirono in tragedia, mentre il terzo, meglio organizzato, avrà successo e rileverà e scandaglierà a fondo il bacino del Mar Morto ${ }^{18}$.

\subsection{Christopher Costigan}

Il primo a tentare l'impresa fu l'irlandese Costi$\operatorname{gan}^{19}$ (1810-1835), che a fine agosto del 1835, dopo aver acquistato una barca a Beirut e aver ingaggiato un marinaio maltese, navigò fino ad Acco, dove caricò la scialuppa su cammelli per trasportarla fino a Tiberiade, da dove cominciò il suo viaggio di esplorazione del Giordano e del Mar Morto. Ben presto dovette desistere dall'impresa di navigare il fiume, dal momento che le acque in quella stagione erano difficilmente navigabili e piene di insidiose rapide. Dopo essere tornato in modo rocambolesco a Gerusalemme, ritorna sulle sponde del Mar Morto e si imbarca assieme al marinaio maltese per riprendere la sua esplorazione. Dopo aver remato girovagando per otto giorni da una sponda all'altra e

\footnotetext{
${ }^{18}$ Non a caso sulla penisola del Lisan il capo a nord e quello a sud portano i nomi di Costigan e di Molineux, che pagarono con la vita il loro tentativo di esplorare il Mar Morto. Porta invece il nome di Lynch lo stretto che passava tra la stessa penisola e la costa occidentale, oggi del tutto asciutto. Per unattenta ricostruzione delle esplorazioni della valle del Giordano e del Mar Morto si vedano Goren, 2004; 2011; 2016.

${ }^{19}$ Le vicende del suo viaggio d'esplorazione lungo il fiume Giordano e sul Mar Morto e la sua tragica fine sono raccontate da Ernest W. G. Masterman in un articolo del 1911, dove raccolse le testimonianze e racconti fatti da vari testimoni a partire da quanto raccontato dal marinaio maltese, che sopravvisse alla spedizione e da T. Nicolayson, che soccorse e assistette Costigan fino alla morte (Masterman 1911, 13-19; O’Loughlin 2011).
}

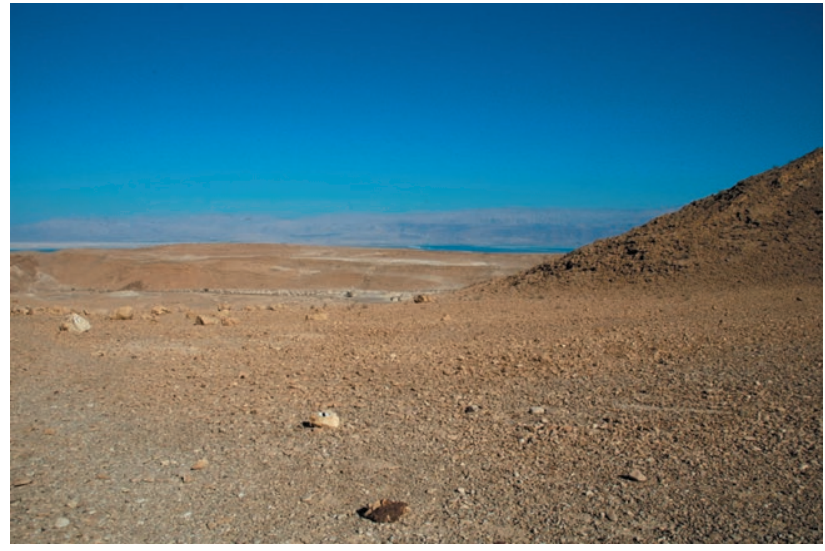

Figura 8. La parte meridionale del Mar Morto vista da occidente (foto dell'autore).

cercando di misurare la profondità delle acque, spossati dal caldo, rimasti senz'acqua potabile e costretti a bere l'acqua insalubre e salmastra del Mar Morto, riescono fortunosamente ad approdare sulla sponda nord occidentale, dove vengono soccorsi da alcuni beduini e trasportati a Gerico.

Mentre il marinaio che era con lui viene mandato a Gerusalemme, Costigan, febbricitante, viene accudito dagli abitanti di Gerico. Finalmente arrivò il Reverendo Nicolayson che si premurò in tutti i modi di far trasportare l'ormai moribondo a Gerusalemme (e per questo meritò l'appellativo di "buon samaritano" nella lapide che la madre fece scolpire per la tomba di Costigan) nella foresteria del Convento di S. Salvatore dei Francescani ${ }^{20}$, dove vani furono i tentativi di farlo rimettere e dove morì nelle prime ore del mattino del sette settembre 1835. Fu sepolto nel cimitero latino del Monte Sion e sulla sua tomba la madre fece apporre una lapide con inciso un toccante epitaffio in latino ${ }^{21}$.

\footnotetext{
${ }^{20}$ Probabilmente la stessa Casa Nova che ospitò il Bajnotti.
}

${ }^{21}$ Siste viator, nec sine lachrymis perlege lapis iste. / Christophorum Costigin III filium Silvestri Costigin Dublinensis tegit iuvenem ingenio miti ac blando patris morientis iussu in collegio hoc Iesu Maynoothwood in Hibernia Lib(e)ralibus disc(i)plinis ad prime excultum / Qui Loca Sancta lustraturus in Mari Asphaltite febre cor(r)eptus indeque a quodam bono samaritano Ierosolymam usque pia cura et labore perductus in Coenobio S. Francisci Sacramentis ecclesiae catholicae rite munitus obiit, / Iustis (exequiis) que ei a PP. solemniter factis Anno Salutis MDCCCXXXV Aetatis XXV / [tralcio di vite] / Filio suo dilectissimo in terra ali(e)na defuncto Pia Mater Catharina / M(emoria) P(erpetua) / Ave Fili Recipiat te coelestis illa Sion ubi nec mors nec luctus nec ullus dolor sed perennis regnat iubilatio. (per la lettura e le integrazioni si rimanda a Piccirillo, 2004). "Fermati viaggiatore, e non senza commozione leggi (ciò che è scritto su) questa pietra. / (Essa) cela Cristoforo Costigan terzo figlio di Silvestro Costigan di Dublino, giovane di indole mite e dolce per ordine del padre morente studiò le scienze liberali in questo Jesus College a Maynoothwood in Irlanda. / 


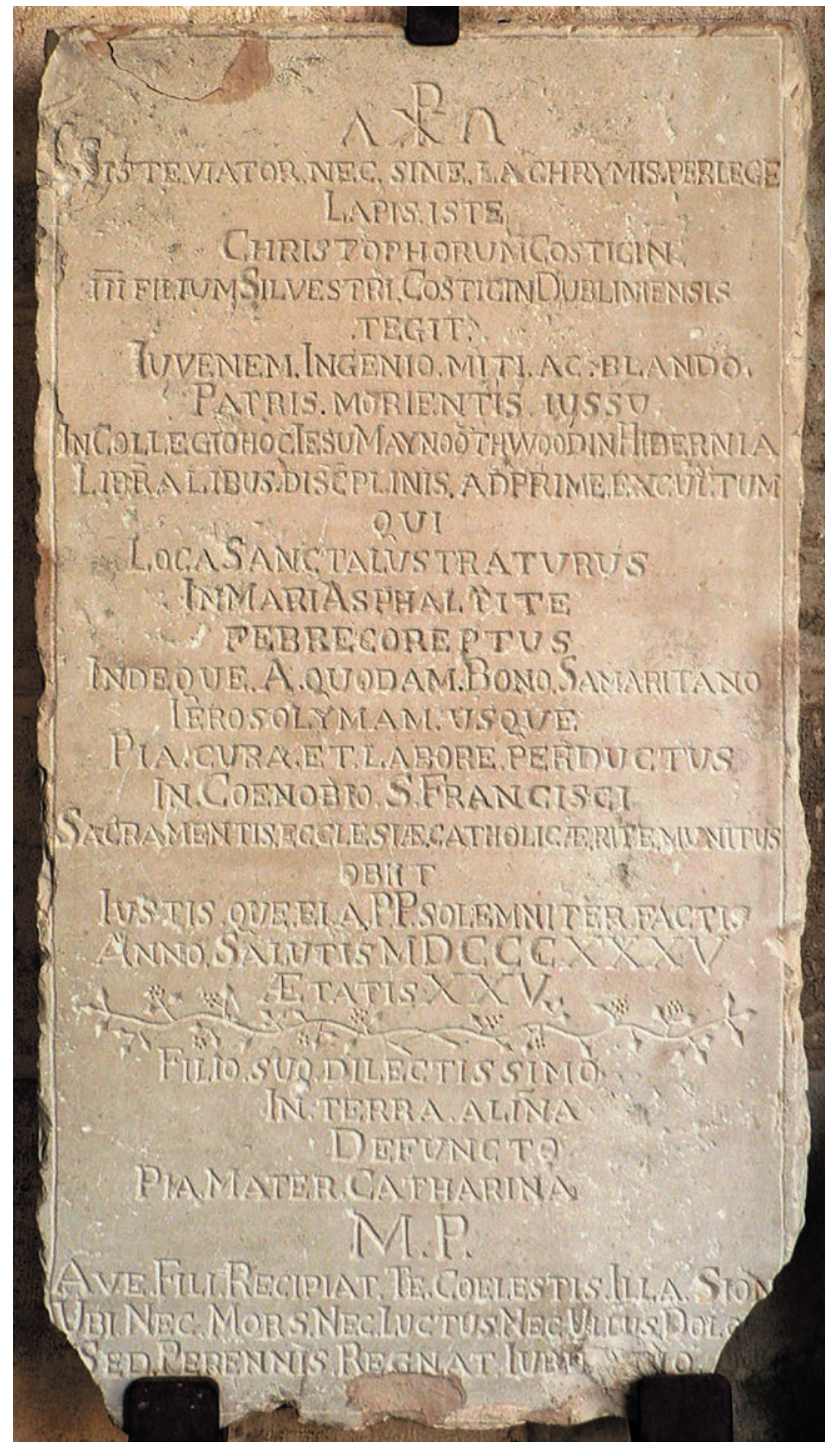

Figura 9. La lapide di Costigan conservata presso il museo della Flagellazione a Gerusalemme (Bsalzberg, CC BY-SA $4.0<\mathrm{https}$ :// creativecommons.org/licenses/by-sa/4.0>, via Wikimedia Commons).

L'esatta ubicazione della tomba col tempo è andata persa, resta invece la lapide conservata dal 2004 negli

\footnotetext{
Accingendosi a visitare i Luoghi Santi fu preso da una febbre presso il Mar Morto e da qua fu portato da un buon samaritano con affettuosa solerzia e sforzo a Gersusalemme nel Convento di S. Francesco. Morì confortato dai Sacramenti della Chiesa Cattolica / e con ogni cosa appropriata compiuta solennemente per lui dai Padri. Nell'anno della salvezza 1835, nel suo 25 esimo anno di vita. / Al suo amatissimo figlio, defunto in terra straniera, la devota madre Caterina / [eresse questo monumento] a perenne memoria. / Ave o figlio, ti accolga quella Celeste Sion dove né morte né lutto né dolore alcuno, ma gioia eterna regna. (trad. di A. Lena).
}

spazi del museo dello Studium Biblicum Franciscanum, presso il convento della Flagellazione ${ }^{22}$.

\subsection{Camille-Antoine Callier}

Il capitano francese Callier (1804-1889), intraprese un viaggio lungo quattro anni (1830-1834) che lo portò in Asia Minore, Siria, Palestina e Arabia Petrea (Callier 1835, 6). Egli ebbe cura di tracciare in modo meticoloso il percorso seguito, come egli stesso fa notare:

\begin{abstract}
"Dans ce pénible et dangereux voyage, j’ai partout dessiné le terrain avec un soin scrupuleux qui donnera une idée exacte de l'aspect physique du désert. Tous les lits de torrens sont indiqués et groupés par bassins, de manière à établir régulièrement le partage des eaux et la direction des pentes" (Callier 1835, 20)
\end{abstract}

La mappa col tracciato del percorso seguito indica che il Callier ha avuto modo di visitare solo la riva nordoccidentale del Mar Morto, mentre lo scopo principale della spedizione organizzata per esplorare l'Arabia Petrea era "d'étudier la partie occidentale de Ouadi el-Ghor, et d'aller vérifier si la bifurcation du golfe Élanite, indiquée par plusieurs géographes, avait une existence réelle" (Callier 1835, 20). Tornerà sul Mar Morto qualche anno dopo con la spedizione del Conte de Bertou.

\subsection{George H. Moore e William George Beek}

L'irlandese Moore (1810-1870) e l'inglese Beek (18041873) nel marzo del 1837 tentano un'impresa simile a quella di Costigan, trasportano una barca da Beirut a Giaffa e da qui al Mar Morto dopo essere passati da Gerusalemme a Gerico. Incominciato un dettagliato survey della costa, vengono fermati dalle autorità locali $\mathrm{e}$ costretti ad interrompere il lavoro intrapreso. Il Bollettino della Royal Geographical Society di Londra riporta, tra l'altro, come "there appears also to be another remarkable feature in the level of the sea, as from several observations upon the temperature of boiling water, it appears to be considerably lower than the ocean" (RGSL 1837), senza però specificare altro, mentre per quel che riguarda la sua profondità annota come in alcuni punti essa raggiunga poco meno di $550 \mathrm{~m}^{23}$.

Difatti, la precisa rilevazione del livello del Mar Morto vide impegnate in quegli anni alcune spedizioni scientifiche internazionali, che utilizzarono diversi meto-

\footnotetext{
${ }^{22}$ Del suo trasferimento dal cimitero al museo ne dà notizia l'allora direttore (Piccirillo 2004).

${ }^{23} 300$ fathoms (RGSL 1837). 1 fathom $=1,8288 \mathrm{~m}$.
} 
di di misurazione (temperatura di ebollizione dell'acqua, barometro, triangolazioni col teodolite) per confermare che la superficie del Mar Morto si trova di alcune centinaia di metri sotto il livello del Mediterraneo.

\subsection{Gotthilf Heinrich von Schubert}

Il naturalista tedesco von Schubert nel 1837 assieme a due suoi giovani assistenti e ad un pittore partì dall'Egitto per raggiungere la Palestina attraverso il Sinai, seguendo il tragitto tracciato da Mosè e dal suo popolo nel racconto dell'Esodo. Dopo essere stati per alcuni giorni a Gerusalemme, scesero a Gerico e da qui raggiunsero la sponda occidentale del Giordano all'altezza del luogo del Battesimo e poi si spostarono sul Mar Morto. Fatte alcune misurazioni barometriche, conclusero anche loro che il livello del Mar Morto fosse più basso di quello del Mar Mediterraneo di circa 195 m (von Schubert 1838 , vol. $3,86-88$ ). La spedizione tedesca e quella inglese si incrociarono sulle sponde del Mar Morto, ma non ebbero modo di scambiarsi informazioni (von Schubert, 1838, vol. 3, 93). Una accesa disputa su chi tra i due gruppi - inglese o tedesco - avesse raggiunto e pubblicato per primo il dato si protrasse per anni, finché non si acclarò che il primato toccasse agli inglesi ${ }^{24}$.

\subsection{Jules de Bertou}

Il francese de Bertou (1809-1881) si recò in Palestina nel 1838 e fu il primo a delineare il bacino idrografico del Wadi Arabah, cercando di dimostrare che il fiume Giordano, attraversato il Mar Morto, sfociasse - attraverso forse un corso sotterraneo - nel Mar Rosso, rifiutando, in un primo momento, il dato ottenuto da Moore e Beek. Si dovette tuttavia ricredere e nella primavera del 1838 realizzò accurate misurazioni barometriche lungo la costa settentrionale e quella meridionale del Mar Morto e a Gerusalemme, che gli permisero di affermare che il Mar Morto era di circa 406 metri al di sotto del livello del Mediterraneo (Callier 1838).

\footnotetext{
${ }^{24}$ Ritter 1866, vol. 3, 127 n. 1. Ritter in questa nota, oltre ad affermare che i primi ad appurare il livello del Mar Morto sono stati Moore e Beek, toglie anche ogni dubbio sul problema del forte dislivello tra Lago di Tiberiade e Mar Morto: "The reader's attention ought to be called particularly to the fact that it is to this expedition that we owe the direct discovery of the depression of the Dead Sea. Robinson cast doubts upon its possibility as recently as 1847 , and the weight of his great authority was a heavy burden to lift. It was proved in the most conclusive manner also by my esteemed friend Dr. Petermann, that so many were the windings of the Jordan between Lake Tiberias and the Dead Sea, that it would require no greater fall per mile than many English rivers exhibit, to reach the supposed depression of the Dead Sea".
}

\subsection{Joseph Ritter von Russegger}

Quella guidata da von Russegger ${ }^{25}$ (1802-1863) è quasi certamente la spedizione "austriaca" a cui fa riferimento Bajnotti nella sua lettera e che si trovava nell'area attorno al Mar Morto nell'autunno del 1838. Russegger eseguì una serie di accurate misurazioni barometriche nel corso del suo viaggio dal Sinai a Nazareth ${ }^{26}$. Nel fare ciò tiene a sottolineare due risultati ottenuti a cui i viaggiatori avrebbero da lì in poi dovuto prestare attenzione: il dislivello del corso del fiume Giordano che si ricava dalle misurazioni del Lago di Tiberiade e del Mar Morto e l'andamento della valle dell'Arabah tra il Mar Rosso e il Mar Morto (Russegger 1841, 187). A differenza di quelli che avevano effettuato la misurazione prima di lui, Russegger ebbe l'opportunità di effettuare le misurazioni varie volte, così da evitare per quanto possibile errori accidentali, registrando i valori prima di 1319 piedi pari$\operatorname{gini}^{27}(428,5 \mathrm{~m})$, e poi di 1341 piedi parigini $(435,6 \mathrm{~m})$ sotto il livello del Mediterraneo (Russegger 1841, 187).

\subsection{John Frederick Anthony Symonds e Edward Aldrich}

Gli inglesi Symonds e Aldrich, furono inviati in Palestina nel 1840 e, dopo aver effettuato rilievi lungo la costa del Mediterraneo, agli inizi del 1841 si recarono a Gerusalemme, dove realizzarono una pianta dettagliata della città. Lo stesso anno vennero incaricati di effettuare un completo survey trigonometrico della parte meridionale della Palestina. Con l'ausilio del teodolite e mediante triangolazioni che andavano dal Mediterraneo al Mar Morto, calcolarono per la superficie di quest'ultimo una quota di $-1311,9$ piedi inglesi $(-399,87 \mathrm{~m})$ sotto il livello del Mediterraneo (Hamilton 1842, LIX-LXI). La misurazione fatta un anno dopo, probabilmente affinata, riporta $-1312,2$ piedi inglesi $(-399,96 \mathrm{~m})$, dati comunque abbastanza in linea tra loro e che confermavano oltre ogni ragionevole dubbio che il Mar Morto, il Lago di Galilea, la valle del Giordano e tutto il wadi Arabah si trovavano ben sotto il livello del Mediterraneo, dal momento che le misurazioni derivanti dall'ebollizione

\footnotetext{
${ }^{25}$ Geologo nato a Salisburgo, dal 1836 al 1839 guidò una spedizione nel Nord Africa, Vicino oriente e Asia Minore, i cui risultati, assieme a quelli di altre sue spedizioni Russeger pubblicò in una serie di volumi dal titolo Reisen in Europa, Asien und Afrika: mit besonderer Rücksicht auf die naturwissenschaftlichen Verhältnisse der betreffenden Länder; unternommen in den Jahren 1835 bis 1841.

${ }^{26}$ Tra le misurazioni riportate in una tabella sono da annotare quelle relative a Gerico, al posto dove i pellegrini si immergono nel Giordano, alla superficie del Mar Morto, alla superficie del Lago di Tiberiade (Russegger 1841, 186).

${ }^{27}$ Un piede parigino equivale a $32,484 \mathrm{~cm}$ (Février, s.d).
} 
dell'acqua, dai dati barometrici e da quelli trigonometrici convergevano verso questa conclusione (Hamilton 1842, LIX-LXI; 1843, LXXIV-LXXV) ${ }^{28}$.

Qualche anno dopo in un suo articolo dal titolo "Depression of the Dead Sea and of the Jordan Valley", pubblicato nel The Journal of the Royal Geographical Society of London del 1848, Edward Robinson pone ancora l'attenzione sull'interessante fenomeno del forte dislivello coperto dal fiume Giordano tra il lago di Tiberiade e il Mar Morto, - pari a circa 16,4 piedi per miglio - e fa notare come, fino ad allora:

"The immediate banks of the Jordan... have never been fully traced between the two lakes, though travellers have passed along the whole length of the valley... In the absence, therefore, of all further observations, and in view of the striking anomaly thus presented by the Jordan as respects all other like rapid streams, I venture to suggest... whether, after all, there may not be a possibility that some slight element of defect or inaccuracy may have entered into the observations or calculations, and thus have affected the correctness of the result?" (Robinson 1848, 79-84).

Cerca così di risolvere il problema insinuando la possibilità di un errore di misurazione sui livelli del lago di Tiberiade e del Mar Morto, senza tener in considerazione - né lui, né tanto meno Hamilton prima di lui la possibilità che in realtà il fiume, salvo alcune rapide, scorresse abbastanza dolcemente, avendo un andamento a meandri, come si sarebbe da lì a breve appurato ${ }^{29}$.

\footnotetext{
${ }^{28}$ Hamilton fa anche notare che, assunto ormai il livello sia del Lago di Tiberiade che quello del Mar Morto, "for it follows from these two ascertained levels that there is a difference of nearly 1000 feet between the Lake of Tiberias and the Dead Sea, a distance in direct line of little more than one degree of latitude, which implies (the Jordan not being a meandering stream) a fall of more than 16 feet in every mile of its course. This is in itself a very remarkable phenomenon, and calls for the early attention of travellers and geographers. The river has been frequently crossed, and is always noted as a rapid stream, but no cataracts or decided rapids as such have been observed, and no one has traced its banks from one of these points to the other. It is earnestly to be hoped that the attention of our engineer officers... will be directed to this remarkable circumstance" (Hamilton 1843, LXXV).

${ }^{29}$ I dubbi sulla correttezza delle misurazioni e di conseguenza il problema della caduta e della pendenza del fiume Giordano continuarono ad essere sollevati per molti anni ancora, benché già nel 1848 ai dubbi sollevati da Robinson (Robinson 1848) risponda Petermann (Petermann 1848) concludendo che: "Thus with regard to the Jordan, although not a meandering river, and forming almost a straight line from the Lake of Tiberias to the Dead Sea, yet the few bends and windings of the river, when taken into account, give it a length of 80 English miles, which is 11 miles longer than Professor Robinson's statement of 60 geographical miles. The 14.3 feet of fall per mile which he thus calculates, will be reduced by the 80 English miles, to 12.3 feet. And I have no doubt that when the course of the Jordan is thoroughly explored, it will exhibit a still greater development, and the rate of fall will be still more reduced" (Peterman 1848, 94). In realtà prima Molyneux e poi Lynch, in quegli
}

La questione aveva sollevato molto interesse in ambito scientifico internazionale, tant'è che anche geografi del calibro di Alexander von Humboldt e di Carl Ritter si interessarono del Mar Morto e della valle del Giordano e delle problematiche ad essi relative. Si devono a quest'ultimo, che riceveva regolarmente relazioni dalle spedizioni in loco sia quella di von Shubert che quella di von Wildenbruch, un volume sul fiume Giordano e il Mar Morto (Ritter 1850) e un'opera in quattro volumi sulla geografia della Palestina e della penisola del Sinai (Ritter 1866).

\subsection{Anton Albert Heinrich Louis von Wildenbruch}

Il prussiano von Wildenbruch (1803-1874), fu console generale in Siria, con sede a Beirut, dal 1829. Di formazione geografo, allievo di Ritter, Wildenbruch cominciò a viaggiare per la Siria e l'Asia Minore, inviando a Berlino, dove rientrò nel '36, dettagliati rapporti sulle sue osservazioni e scoperte in territori fino ad allora poco conosciuti. Ottenuto un secondo incarico nella stessa sede dal ' 42 al '48, continuò le sue ricerche, inviando regolarmente dei rapporti sia alla Gesellschaft für Erdkunde zu Berlin, che alla Royal Geographical Society di Londra (Ritter 1846; Wildenbruch 1850). Le sue misurazioni barometriche erano particolarmente accurate, perché costantemente confrontate con le misurazioni di un secondo barometro allineato al livello del Mediterraneo. Per quello che qui interessa, il lago di Tiberiade viene dato a $-793,3$ piedi parigini $(-257,70 \mathrm{~m})$, mentre il Mar Morto a $-1351,6$ piedi parigini $(-439,05 \mathrm{~m})^{30}$.

\subsection{Thomas Howard Molyneux}

A poco più di dieci anni dal tragico tentativo di Costigan, il tenente della Regia Marina inglese Molyneux (1801-1847) (Molyneux 1848; cfr. anche Robinson 1848) organizzò una nuova spedizione per esplorare la valle del Giordano e in particolare navigare sul Mar Morto. Anche lui, come Costigan, arrivò ad Haifa da Beirut sulla nave britannica Spartan, da qui il 20 agosto del 1847 su di una scialuppa si trasferì, assieme a tre marinai volontari e una guida di Beirut, ad Acco, da dove proseguì trasportando la scialuppa via terra fino a Tiberiade. L'intento del suo viaggio, come esplicitamente detto all'inizio del suo resoconto

stessi anni, esplorando il corso del fiume Giordano ne registrano la tortuosità, facendo venir meno il principale argomento col quale si sosteneva l'erroneità o la forte imprecisione delle misurazioni del livello del Mar Morto e del Lago di Tiberiade.

${ }^{30}$ Non viene esplicitato da nessuna parte che si tratti di piedi parigini, ma viene dato per scontato in Goren 2004, 155. 


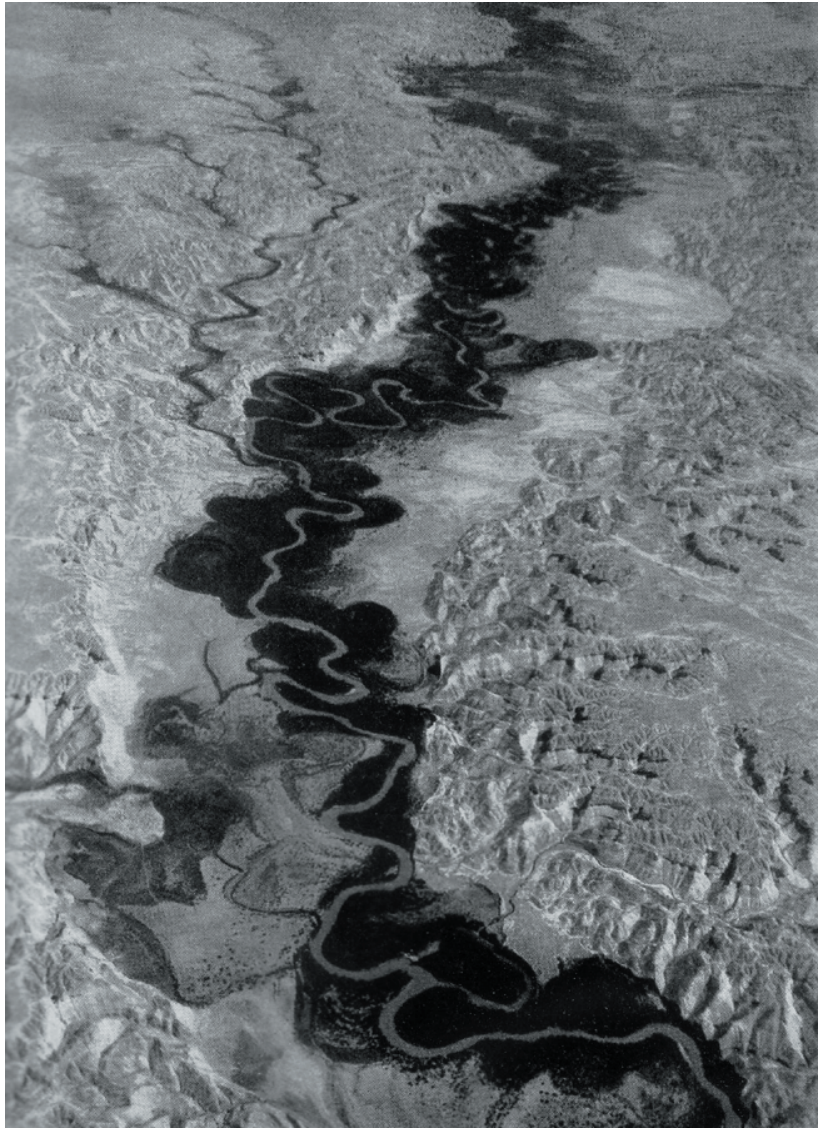

Figura 10. Le anse del fiume Giordano (W. Robert Moore - The National Geographic Magazine, Dec. 1938; Public domain).

pubblicato sul The Journal of the Royal Geographical Society dell'anno successivo, era quello di navigare lungo il Giordano fino al Mar Morto con l'obiettivo di esaminare il corso del fiume, esplorare la valle attraverso cui scorre e specialmente misurare la profondità del Mar Morto ${ }^{31}$. Una volta fatto ciò avrebbe riportato la scialuppa alla nave, portandola via terra prima a Gerusalemme e poi a Giaffa. Dopo aver esplorato il lago di Tiberiade, misurandone l'ampiezza e la profondità, procede verso sud lungo il Giordano. Il viaggio si fa subito difficile, data l'impraticabilità del fiume che in molti punti, a causa anche della poca acqua nella stagione estiva, risulta impossibile da navigare o guadare, al punto che più volte Molyneux e i suoi si videro costretti a tirare in secco la barca e caricarla sui cammelli, per proseguire a discendere verso il Mar Morto lungo le rive del fiume, il cui corso, che scorre tortuoso

\footnotetext{
31 "Our objects were to examine the course of the Jordan, as well as of the valley through which it runs, and specially to measure the depth of the Dead Sea" (Molyneux 1848, 104).
}

tra la vegetazione lussureggiante, richiama "un gigantesco serpente che si snoda lungo la valle" 32 .

Il viaggio a tappe cominciato sulle sponde del lago di Tiberiade il 23 agosto proseguirà lungo la valle del Giordano fino al 31, quando gli esploratori arrivano a Gerico. Il 3 settembre, messa la barca in acqua presso la foce del Giordano, alle 6 del pomeriggio Molyneux con solo un paio di remi e con delle persone senza la minima esperienza di navigazione, prende il largo mentre comincia a farsi buio ${ }^{33}$. Il giorno seguente vengono effettuate alcune misurazioni della profondità del Mar Morto, registrando i dati di 411,5 m, 325,5 m e 334,7 m. Rimasero a misurare la profondità ed osservare le sponde del Mar Morto per quasi due giorni interi durante i quali perlustrarono buona parte del bacino al di sopra della penisola del Lisan, finché, durante la giornata del 5 settembre, risaliti verso nord, non trovarono un punto di approdo presso la foce del Giordano. Molyneux rientra a Gerusalemme il 7 settembre e tre giorni dopo si imbarca sullo Spartan che era all'ancora a Haifa e su di esso fa ritorno a Beirut. Qui però, purtroppo, si ammala, forse fiaccato dalle fatiche dell'escursione, e muore il 4 ottobre del 1847.

\subsection{William Francis Lynch}

La spedizione guidata dall'americano Lynch (18011865) nel 1848 fu quella che meglio ebbe modo di esplorare l'area del Giordano e del Mar Morto alla metà del XIX secolo, raccogliendo dati assai significativi. I risultati della spedizione verranno pubblicati in un volume dato alle stampe l'anno successivo, dal titolo Narrative of the United States' expedition to the river Jordan and the Dead Sea (Lynch 1849). Lallora capitano di corvetta - a cui erano state accordate due imbarcazioni metalliche, una con lo scafo di rame, l'altra in ferro galvanizzato - era a capo di una spedizione composta da altri tredici membri dell'equipaggio e da due altre persone che si unirono volontariamente al gruppo, uno a Costantinopoli, l'altro a Beirut. Salpato da Brooklyn il 20 novembre del 1847 a bordo della nave Supply giunge a Beirut il 25 marzo dell'anno successivo. Dalla città libanese si trasferisce ad Acri e da qui comincia il viaggio di esplorazione vero e proprio. Dopo aver visitato la Galilea, Lynch arriva sul Lago di Genezaret all'altezza di Tiberia-

32 “...its winding course, which was marked by luxuriant vegetation, looked like a gigantic serpent twisting down the valley" (Molyneux 1848, $111)$.

33 "We shoved of from this vile place, just as it was falling dark, with only two oars, and with no one who had much idea of using them, except myself, or any notion of boat-sailing" (Molyneux 1848, 126). 


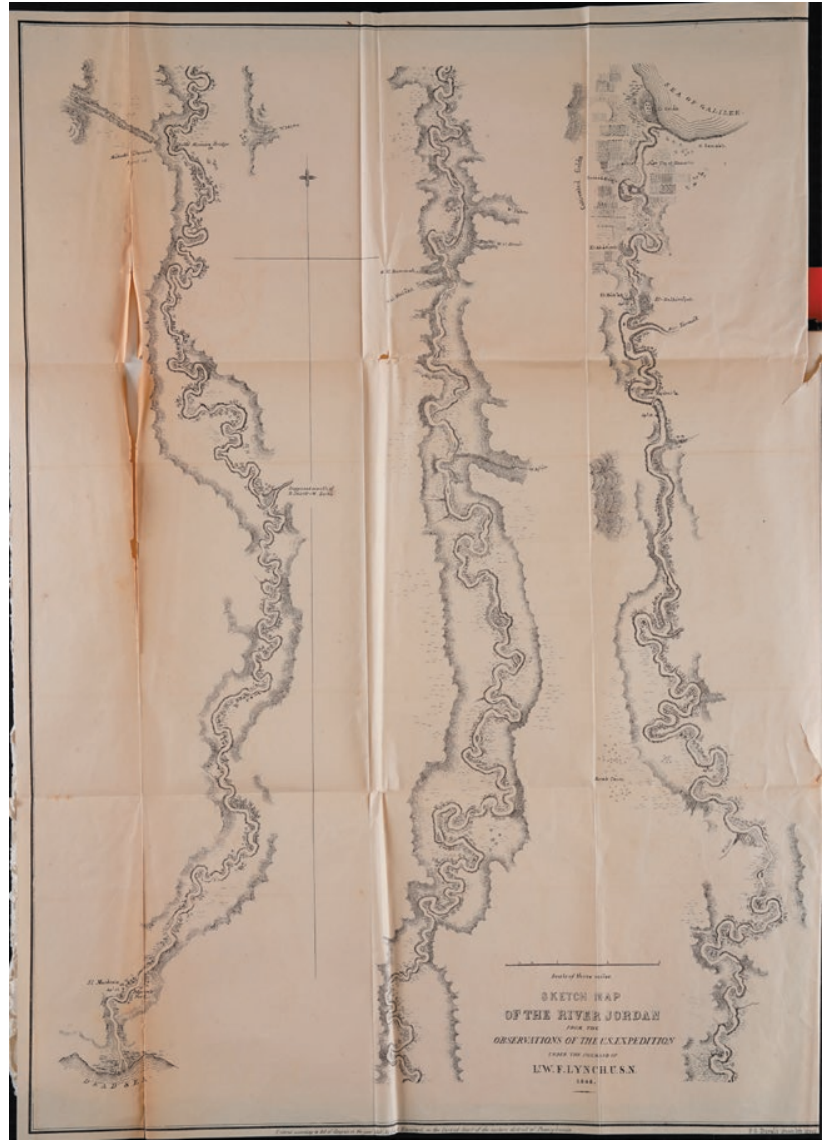

Figura 11. Mappa del fiume Giordano (Lynch 1848, Public domain).

de e, messe in acqua le barche, ne esplora rapidamente la sponda occidentale risalendo fino a Mejdel o Magdala e ne misura la profondità di 27,5 fathoms pari a 165 piedi $(50,3 \mathrm{~m})^{34}$. Con non poche difficoltà il gruppo comincia la discesa lungo il fiume Giordano, che si rivela da subito difficile da navigare e pericoloso per la presenza di alcune rapide. Il dover affrontare il trasferimento verso il Mar Morto quasi sempre via terra, ora sulla sponda occidentale, ora su quella orientale, permette agli esploratori di meglio descrivere e rilevare la valle del fiume e i suoi tortuosi meandri, nonché di spingersi anche nell'entroterra per visitarne alcune località più importanti, come ad esempio Gadara o Gerico. Il 18 aprile 1848, arrivano in prossimità del Mar Morto. Qui Lynch redige un breve rapporto del viaggio lungo la valle del Giordano, dove

${ }^{34} \mathrm{~A}$ tale proposito precisa: "The bottom is a concave basin, - the greatest depth, thus far ascertained, twenty-seven and a half fathoms (165 feet); but this inland sea, alternately rising and falling, from copious rains or rapid evaporation, apart from its only outlet, is constantly fluctuating in depth" (Lynch 1849, 165).

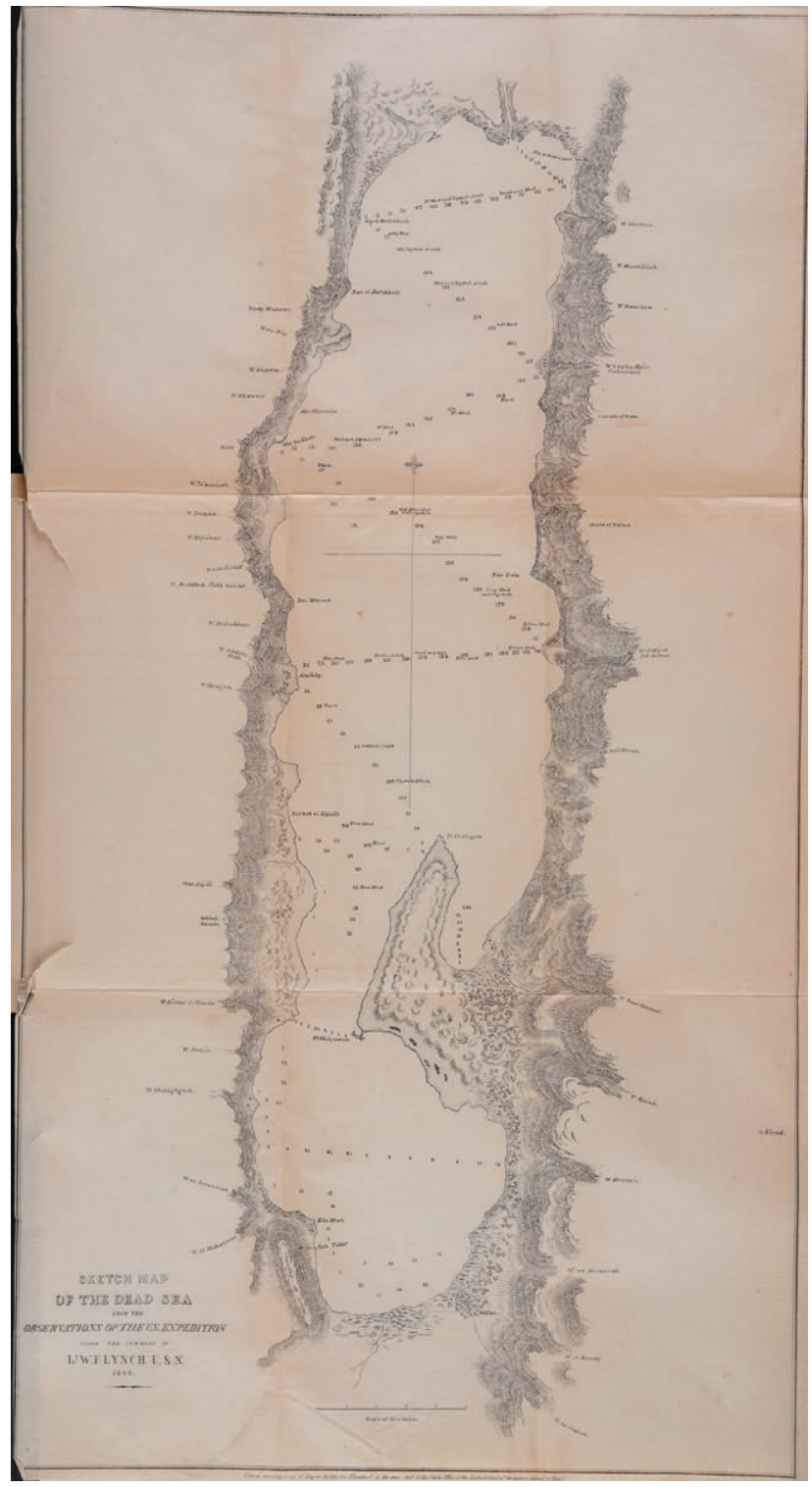

Figura 12. Mappa del Mar Morto (Lynch 1848, Public domain).

tra l'altro scrive che il corso del fiume è molto tortuoso, perfino più del Mississippi ${ }^{35}$.

Arrivati ad Ayn Fishkah mettono le barche in acqua e cominciano a scandagliare i fondali del Mar Morto, registrando le varie profondità e le caratteristiche: i dati

35 "The great secret of the depression between Lake Tiberias and the Dead Sea, is solved by the tortuous course of the Jordan. In a space of sixty miles of latitude and four or five miles of longitude, the Jordan traverses at least 200 miles. The river is in the latter stage of a freshet - a few weeks earlier or later, and passage would have been impracticable. As it is, we have plunged down twenty-seven threatening rapids, besides a great many of lesser magnitude... its course is more sinuous even than of the Mississippi" (Lynch 1849, 265). 


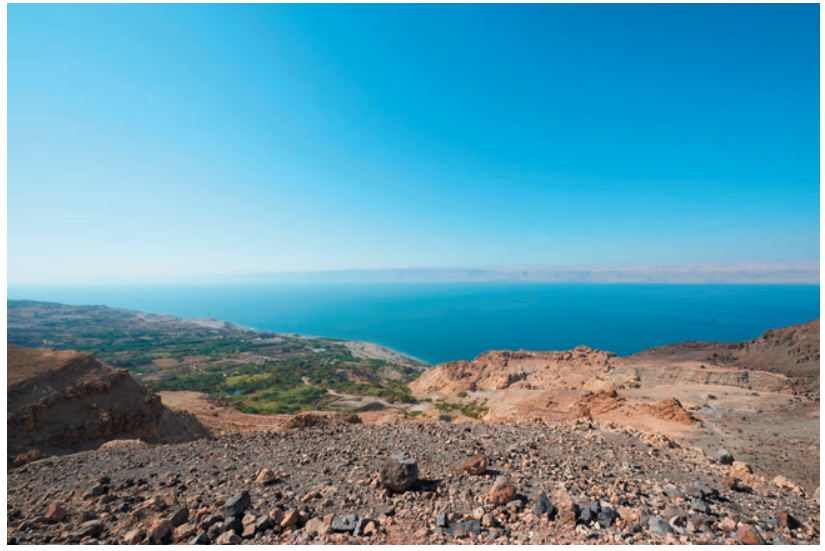

Figura 13. Il Mar Morto dall'altopiano sopra Calliroe (foto dell'autore).

poi verranno riportati nella pianta allegata al volume ${ }^{36}$ e nella dettagliata relazione ufficiale pubblicata nel 1852 (Lynch, 1852).

Tra le annotazioni del 20 aprile viene riportata la differenza di livello del Mar Morto rispetto al Mediterraneo, ottenuta mediante misurazione barometrica, di 1234,589 piedi (se piedi inglesi corrispondono a $376,12 \mathrm{~m}$, se piedi francesi $401,04 \mathrm{~m})^{37}$. Dopo aver esplorato la parte meridionale e le sue coste, Lynch e i suoi risalgono fino ad Ein Gheddi, in seguito visitano Masada e poi si recano sulla sponda orientale, percorrendola quasi in tutta la sua lunghezza, salendo fino a Kerak, e arrivando fino alle acque termali di Calliroe. È il 10 maggio quando Lynch e i suoi uomini lasciano l'accampamento sulle rive del Mar Morto per risalire alla volta di Gerusalemme. Dopo aver visitato la Città Santa si trasferiscono a Giaffa, da qui a Nazaret per poi andare a Damasco, passando per le fonti del Giordano e da Damasco a Beirut, dove il 30 luglio si imbarcano sulla nave francese La Perle d'Orient alla volta di Malta da dove, dopo una quarantena, il 12 settembre si imbarcano sul Supply in direzione dell'America.

\subsection{Carel Willem Meredith van de Velde}

Il cartografo e disegnatore olandese van de Velde (1818-1898) si reca in Palestina tra il 1851 e il 1852. Qui effettua diversi survey, rilievi e disegni che lo porteranno

\footnotetext{
${ }^{36}$ Si tratta della prima mappa accurata del fiume Giordano e del Mar Morto. Cfr. Lynch 1849, 13 e 269.

${ }^{37}$ Lynch 1852, 32. Nel suo rapporto Lynch non specifica, e si sarebbe portati a pensare che si tratti di piedi inglesi, tuttavia come fatto da Goren 2004, 156, che riporta la misura di 401,24, se si considerano piedi francesi la misurazione sarebbe molto più vicina a quello che doveva essere il livello del Mar Morto a quel tempo.
}

a pubblicare nel 1858 una dettagliata mappa della regione, ricavata - come egli stesso annota sulla carta stessa - oltre che dai suoi rilievi anche mettendo assieme i dati disponibili dalle missioni che lo avevano preceduto ${ }^{38}$. Sulla carta, redatta su 8 fogli in scala 1:315000, oltre alla mappa della Palestina vengono riportate anche due piante di Gerusalemme e dei suoi dintorni e, soprattutto, quattro sezioni altimetriche; in esse viene riportato il livello del Lago di Tiberiade (-653 piedi inglesi $=-199,03 \mathrm{~m})$ e quello del Mar Morto $(-1317$ piedi $=-401,42 \mathrm{~m})$. Van de Velde aveva già pubblicato nel 1854 anche un dettagliato resoconto in due volumi del suo viaggio in Siria e Palestina, con un'ampia parte dedicata all'escursione nella regione del Mar Morto e della Giudea meridionale (van de Velde 1854, vol. 2, 1-148).

\subsection{Heinrich Kiepert}

Il cartografo tedesco Kiepert (1818-1899) (Engelman 1977) produsse una prima carta della Palestina per i tre volumi Biblical Researches in Palestine and Adjacent Countries pubblicati da Edward Robinson e Eli Smith prima nel 1841 e poi nel 1856. Nel 1869 fu invitato alla cerimonia di apertura del canale di Suez. Per ragioni finanziarie, tuttavia, la partenza venne rinviata, dal momento che Kiepert intendeva approfittarne per visitare anche la Palestina e l'Asia Minore. La spedizione, - di cui facevano parte, oltre a Kiepert, suo figlio Richard e il medico Paul Langerhans - partì comunque nella primavera del $1870^{39}$, ma si dovette interrompere bruscamente l'anno successivo a causa della guerra con la Francia. Ebbe comunque modo di esplorare per tre settimane la regione ad oriente del Giordano e del Mar Morto.

\subsection{Henry Baker Tristram}

Il prelato anglicano Tristram (1822-1906) fu un viaggiatore, ornitologo e biblista, che si recò più volte in Palestina tra il 1858 e il 1881 ed ebbe modo di studiare dettagliatamente quella regione, pubblicando nel 1865 il volume The land of Israel; a journal of travels in Palestine (Tristram 1865) e nel 1873 Land of Moab: travels and discoveries on the east side of the Dead Sea and the Jordan (Tristram 1873). Porta, inoltre, la sua firma il volume del

\footnotetext{
${ }^{38}$ La legenda sulla carta del 1858 riporta: "Map of the Holy Land constructed by C. W. M. Van de Velde, ... from his own surveys in 1851 \& 1852, from those made in 1841 by Majors Robe and Rochford Scott, Lieut. Symonds and other officers of her Majesty's Corps of Royal Engineers; and from the results of the researches made by Lynch, Robinson, Wilson, Burckhardt, Seetzen etc. 1858".

${ }^{39}$ A questa spedizione fa riferimento il Negri nel suo discorso alla Seduta della SGI di quello stesso anno (supra).
} 


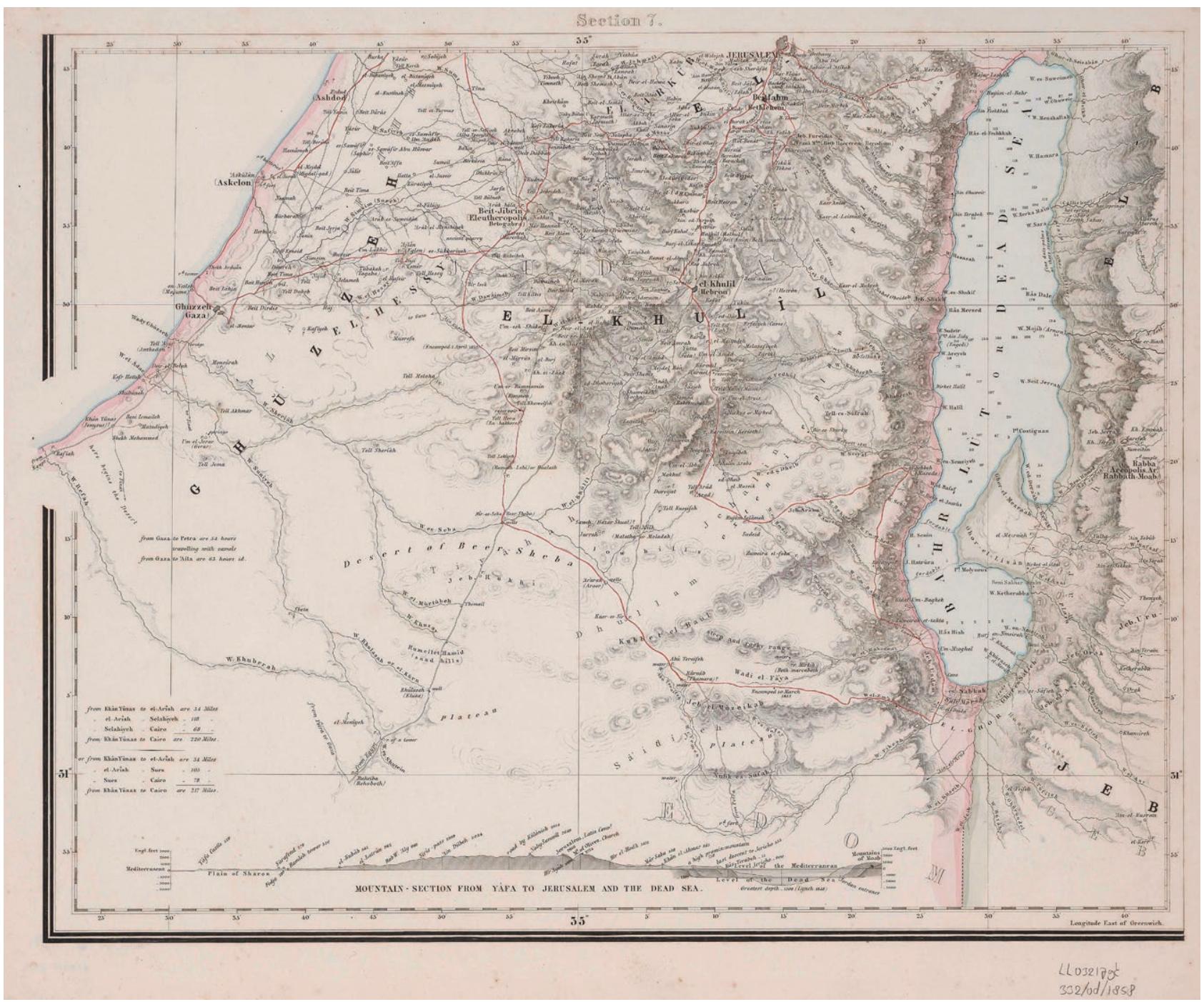

Figura 14. Il foglio n. 8 della carta di van de Velde con il Mar Morto e la sezione dal Mediterraneo al Mar Morto (1858; NKC 1.0).

1885 The Fauna and Flora of Palestine, quarto volume del Survey of Western Palestine a cura del Palestine Exploration Fund. In entrambi i primi due volumi Tristam si sofferma a descrivere il Mar Morto e il suo paesaggio.

6.17 Il Palestine Exploration Fund e le rilevazioni della seconda metà dell' 800 e inizi '900

Con la costituzione del Palestine Exploration Fund (PEF) nel 1865, la prima istituzione fondata con lo scopo precipuo di studiare in maniera scientifica e sistematica il territorio della Palestina ${ }^{40}$, anche le misurazioni

${ }^{40}$ In realtà un survey accurato della regione era già stato fatto dagli ingegneri militari inglesi negli anni '40-41. Cfr. Jones, 1973. Per un excursus del livello del Mar Morto e delle sue fluttuazioni diventeranno più precise (Underhill 1967). È uno dei soci fondatori del PEF, Charles W. Wilson, che si occuperà di ciò. Come egli stesso annota, la prima misurazione fatta il 12 marzo 1865, ammontava a $-1292,13$ piedi $(-393,84 \mathrm{~m})$ (Wilson 1900, 368). Alcuni anni dopo, nella primavera del 1874, nel tentativo di tenere sotto controllo il livello del Mar Morto, Claude R. Conder e Charles F. Tyrwhitt-Drake piazzeranno dei pali graduati (Drake 1874, 187), che verranno però rimossi o trafugati entro pochi giorni. Sarà solo a partire dal 1900 che cominceranno ad essere effettuate delle misurazioni

sulle esplorazioni e la cartografia di Palestina del XIX secolo cfr. Goren 2002. 
continue e costanti delle fluttuazioni di livello del Mar Morto. È G. Hill a sollevare la questione della mancanza di un monitoraggio costante del livello del Mar Morto, partendo dal notare l'inusuale innalzamento di tale livello negli ultimi anni dell'800, facilmente riscontrabile anche ad occhio nudo ${ }^{41}$. Per ovviare alla mancanza evidenziata da Hill e per cercare una risposta agli interrogativi posti, Wilson - assai scettico a riguardo di un'eventuale attività sismica - auspica che venga costantemente monitorato il livello sia del Lago di Tiberiade, che del Mar Morto. Per quest'ultimo propone che si incida un segno su di una roccia presso 'Ain Feshka a 8 o 10 piedi sopra il livello del Mare e che da questo punto vengano periodicamente rilevate le variazioni ${ }^{42}$. Ernest W. Masterman assume l'incarico di effettuare lungo il corso dell'anno le misurazioni, che vengono pubblicate nel Palestine Exploration Quarterly, che nel numero del 1913 contiene un resoconto complessivo a partire dai dati raccolti in cui si legge:

"It is clear, as has been suggested above, that the rainfall has much to do with the varying level... we have indubitable evidence of a considerable rise between 1850 and 1892; and it is interesting to notice that, since the regular observations of the level have been begun, the rise, with fluctuations, has continued' (Masterman 1913, 195-197).

Un altro resoconto sull'andamento del livello del Mar Morto uscirà alla fine del 1935, dove si evidenzia come, stando ai dati raccolti, "it would appear that the level has fallen considerably in those twenty-three years" (Salmon, McCaw 1936, 104).

Da notare come uno dei punti a cui anche i membri del PEF si riferiscono per le rilevazioni del livello del Mar Morto, è quello di Rujm al-Bakar, un isolotto con delle rovine che si trova sulla sponda nordocciden-

41 "I have not observed any reference in the Quarterly Statement to the fact that the surface of the Dead Sea has risen considerably of late years" (Hill 1900, 273). Più avanti si chiede se questo non possa dipendere da qualche movimento di origine tettonica del fondo del Mar Morto, piuttosto che dall'afflusso di acqua, dal momento che quell'anno non era stato particolarmente piovoso. "Is it possible that there is some volcanic action at work raising the bed of the lake? There was a slight earthquake here in January last, which was also felt as far off as Tiberias and Safed. And I may here record a strange appearance which we beheld from this house in May, 1899, and which may indicate volcanic action" (Hill 1900, 276).

42 "The subject is of so much interest that I hope steps may be taken to obtain observations of the level of the Sea of Galilee and of the Dead Sea at different periods of the year... In the case of the Dead Sea I would suggest that a mark be cut on the rocks near 'Ain Feshka, or at some convenient point, at a measured height of 8 or 10 feet above the level of the water on November 1st, when it is at its lowest, and that the level of the water with reference to this mark be measured at the end of each month of the rainy season" (Wilson 1900, 368).

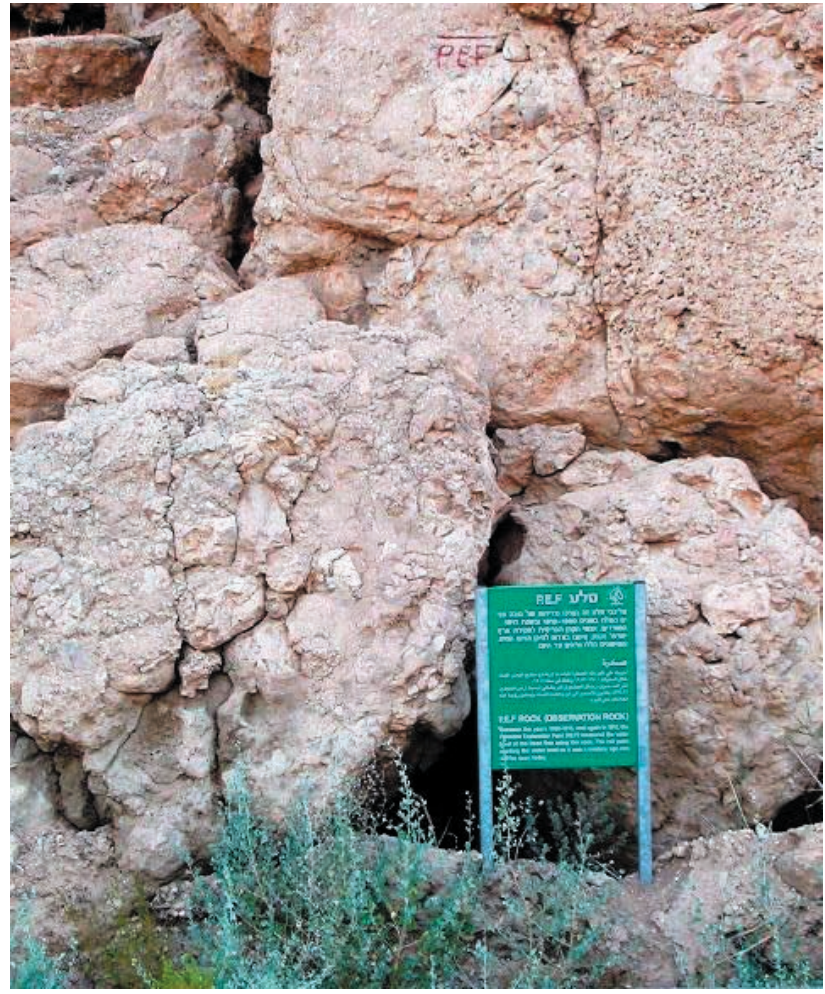

Figura 15. Il marchio di riferimento usato dal PEF dal 1900 in poi per misurare le variazioni di livello del Mar Morto (Public domain).

tale del Mar Morto, non lontano dalla foce del Giordano, che, a seconda del livello del mare, era a volte raggiungibile a piedi a volte no, e che a causa dell'innalzamento del livello dell'acqua negli ultimi anni del XIX secolo e gli inizi del XX era del tutto sommerso. A quest'isolotto faceva riferimento fra Liévin de Hamme, nella nota succitata, arrivando a concludere che il livello del mare stesse risalendo nel corso della seconda metà del XIX secolo.

\section{Conclusioni}

Per concludere questo excursus, è importante notare come, verso la metà degli anni ' 30 del XX secolo, dopo circa un secolo di esplorazioni e misurazioni, il livello del Mar Morto - ormai assodato che fosse ben al di sotto di quello del Mediterraneo - è tenuto sotto controllo e le sue variazioni sono riconducibili quasi esclusivamente all'apporto d'acqua dal Giordano e dagli altri torrenti stagionali che vi sfociano e dalla forte evaporazione dovuta al caldo. Niente è da ricondurre a fantasiosi collegamenti sotterranei con il Mar Rosso o il Mediterraneo, tantomeno ad una presunta attività vulcanica sommersa. Tuttavia è da 


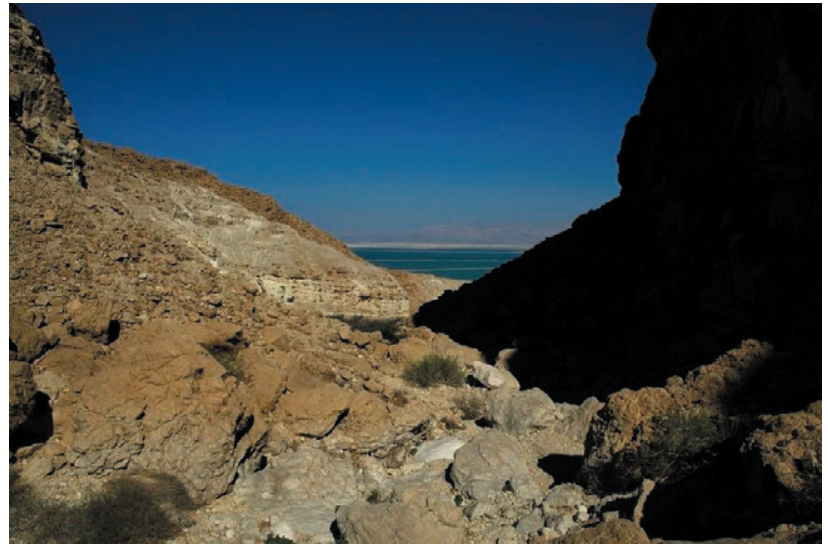

Figura 16. Il Mar Morto visto dal wadi a nord di Jabal Usdum (foto dell'autore).

notare come l'interesse per tutta l'area della depressione del Mar Morto e del Ghor sia uno dei leitmotiv ricorrenti nella moderna storia dell'esplorazione della Palestina fino agli anni a ridosso dell'ultimo conflitto mondiale. Pertanto, la breve nota che Paolo Bajnotti invia a Cristoforo Negri e il conseguente interessamento di quest'ultimo per la questione sollevata da quelle poche righe, ben si inquadrano in questo contesto. L'interesse per la valle del Giordano e per il Mar Morto resterà non sopito nei decenni a seguire tra i geografi italiani, al punto che, nel corso del secondo decennio dello scorso secolo, si cercherà di costituire un "fondo per lo studio geografico della Palestina", la cui prima missione sarebbe dovuta essere proprio quella di condurre approfondite indagini sul Mar Morto. Il progetto, purtroppo, si arenò con l'insorgere delle problematiche di politica internazionale che sarebbero sfociate, da lì a poco, nella seconda guerra mondiale.

\section{Riferimenti bibliografici}

ASCTS (1869). Case Nove. Gerusalemme. Registri degli ospiti. Minute 2.

ASCTS (1874). Case Nove. Gerusalemme. Registri degli ospiti. 4.

Bassi, A. (1857). Pellegrinaggio storico e descrittivo di Terrasanta (3 voll.). Torino, Tipografia subalpina.

Burckhardt, J. L. (1822). Travels in Syria and the Holy Land. London, J. Murray.

Callier, C. (1835). Voyage en Asie Mineure, en Syrie, en Palestine et en Arabie-Pétrée. Bulletin de la Société de Géographie, Douxiéme Sèrie 3/13, 5-22.
Callier, C. (1838). Note sur le voyage de M. le comte de Bertou, depuis le lac Asphaltite jusqu'à la mer Rouge, par le Ouadi-èl-Araba. Bulletin de la Société de Géographie, Douxiéme Sèrie 10/55, 84-100.

Cassini da Perinaldo, F. (1855). La Terra Santa descritta dal P. Francesco Cassini da Perinaldo (3 voll.). Genova, Ferrando.

Conder, C. R., Kitchener, H. H. (1883), The Survey of Western Palestine. Memoirs of the Topography, Orography, Hydrography, and Archaeology. Volume III. Sheets XVIIXXVI. Judea, London, The Committee of the Palestine Exploration Fund.

De Hamme, L. (1887). Guide-indicateur des sanctuaries et lieux historiques de la Terre-Sainte, 3 ed. (2). Jerusalem, Imprimerie des PP. Franciscains.

de Looze, H. (2016). Edouard Colleman (1822-1898) alias Frère Liévin de Hamme. Hamse minderbroeder begeleidde pelgrims in het Heilig Land. Retrieved 15-06, 2021 from https://tincolycklama.files.wordpress.com/2016/06/ edouard-colleman-alias-lic3a9vin-de-hamme-hamseminderbroeder-begeleidde-pelgrims-in-het-heilig-land2016-by-hugo-de-looze.pdf

Drake, T. (1874). Mr. Tyrwhitt Drake's Reports. Palestine Exploration Quarterly, 6(3), 187-190. doi:10.1179/ peq. 1874.018

Engelman, G. (1977). Kiepert, Heinrich. Neue Deutsche Biographie, 11, 593-594 (da https://www.deutsche-biographie.de/pnd116168862.html\#ndbcontent).

Février, D. (s.d.). Une histoire du mètre. Retrieved from https://web.archive.org/web/20110228185545/http://www. industrie.gouv.fr/metro/aquoisert/metre.htm

Frescobaldi, L. (2012). Viaggio di Lionardo di Niccolò Frescobaldi in Egitto e in Terra Santa. Cambridge: Cambridge University Press. doi:10.1017/cbo9781139162630

Gavrieli, I. (2004). The Evolution of the Dead Sea, Past Present and Future, and the Proposed "Peace Conduit". Horizons in Geography, 60-61, 163-165. doi: $10.2307 / 23712693$

Goren, H. (2002). Sacred, but Not Surveyed: NineteenthCentury Surveys of Palestine. Imago Mundi, 54, 87-110. doi: $10.2307 / 1151507$

Goren, H. (2004). How Low is the Lowest Point on Earth? The Story of Determining the Level of the Dead Sea. Horizons in Geography, 60-61, 147-162. doi:10.2307/23712692

Goren, H. (2011). Dead Sea Level: Science, Exploration and Imperial Interests in the Near East (6). London, Tauris. 
Goren, H. (2016). Geography of religious traditions. The beginning of scientific study of the Dead Sea. Negev, Dead Sea and Arava Studies, 8 (1), 3-16.

Hamilton, W. R. (1842). Address to the Royal Geographical Society of London. The Journal of the Royal Geographical Society of London, 12, xxxv-lxxxix. doi:10.2307/1797975

Hamilton, W. R. (1843). Address to the Royal Geographical Society of London. The Journal of the Royal Geographical Society of London, 13, xli-cv. doi:10.2307/1798131

Hill, G. (1900). The Dead Sea. Palestine Exploration Quarterly, 32 (3), 273-282. doi:10.1179/peq.1900.32.3.273

Irby, C. L., Mangles, J. (1823). Travels in Egypt and Nubia, Syria, and Asia Minor, during the years 1817 and 1818. Cambridge: Cambridge University Press.

Jones, Y. (1973). British Military Surveys of Palestine and Syria 1840-1841. The Cartographic Journal, 10 (1), 29-41. doi:10.1179/caj.1973.10.1.29

Klein, C., Flohn, H. (1987). Contributions to the knowledge of the fluctuations of the Dead Sea level. Theoretical and Applied Climatology, 38(3), 151-156. doi:10.1007/bf00868099

Klimas, N. S. (2015). La Casa Nova di Gerusalemme. Lospizio dei pellegrini. Frate Francesco, 81 (1), 197-218.

Kreiger, B. (2016). The Dead Sea and the Jordan River. Retrieved from http://books.google.it/books?id=DfrpsgE ACAAJ\&hl=\&source $=$ gbs_api

Lando, F. (2012). La geografia umanista: un'interpretazione. Rivista Geografica Italiana, 119, 259-289.

Lichtenberger, A. (2011). Seetzen, Ulrich Jasper. In Das wissenschaftliche Bibellexikon im Internet (WiBiLex). Deutsche Bibelgesellschaft. Retrieved from https://www. bibelwissenschaft.de/stichwort/15561/

Lynch, W. F. (1849). Narrative of the United States' expedition to the river Jordan and the Dead Sea. Philadelphia, Lea and Blanchard.

Lynch, W. F. (1852). Official Report of the United States' Expedition to Explore the Dead Sea and the River Jordan. Baltimore, John Murphy.

Maggioli, M. (2013). Negri, Cristoforo. In Dizionario Biografico degli Italiani. Retrieved from https://treccani.it/ enciclopedia/cristoforo-negri_\%28Dizionario-Biografico\%29/

Mahlmann, W. (1846). Russegger's Bemerkungen über dessen meteorologische Höhenbestimmung des Todten Meers, datirt Hall in Tirol, am 4. September 1845.
Monatsberichte über die Verhandlungen der Gesellschaft für Erdkunde zu Berlin, 7, 163-168.

Masterman, E. W. G. (1905). Explorations in the Dead Sea Valley. The Biblical World, 25(6), 407-417. doi: $10.2307 / 3140564$

Masterman, E. W. G. (1911). Three Early Explorers of the Dead Sea Valley: Costigan - Molyneux - Lynch. Palestine Exploration Fund Quarterly Statement, 43 (1), 12-27.

Masterman, E. W. G. (1913). Summary of the Observations on the Rise and Fall of the Level of the Dead Sea, 1900-1913. Palestine Exploration Quarterly, 45(4), 192197. doi:10.1179/peq.1913.45.4.192

Molyneux, T. H. (1848). Expedition to the Jordan and the Dead Sea. The Journal of the Royal Geographical Society of London, 18, 104-130. doi:10.2307/1797937

Negri, C. (1870). Adunanza solenne della Società tenuta il 13 marzo 1870. Discorso. Bollettino della Società Geografica Italiana, 4, 1-64.

O’Loughlin, T. (2011). “The Unfortunate Costigan”, First Surveyor of the Dead Sea. History Ireland, 19,1, 24-26.

Petermann, A. (1848). On the Fall of the Jordan, and of the Principal Rivers in the United Kingdom. The Journal of the Royal Geographical Society of London, 18, 89-104. doi: $10.2307 / 1797936$

Piccirillo, M. (2004). Museo dello SBF, nuove acquisizioni. Notiziario Studium Biblicum Franciscanum Jerusalem Anno Accademico, 2003-2004 (p. 24).

RGSL. (1837). On the Dead Sea and Some Positions in Syria. The Journal of the Royal Geographical Society of London, 7, 456. doi:10.2307/1797544

Ritter, C. (1846). L. v. Wildenbruch's Profilzeichnungen nach barometrischen Nivellements in Syrien. Monatsberichte über die Verhandlungen der Gesellschaft für Erdkunde zu Berlin, 7, 270-272.

Ritter, C. (1850). Der Jordan und die Beschiffung des Todten Meeres. Berlin: G. Reimer.

Ritter, C. (1866). The Comparative Geography of Palestine and Sinaitic Peninsula (4 voll.).

Robinson, E. (1848). Depression of the Dead Sea and of the Jordan Valley. The Journal of the Royal Geographical Society of London, 18, 77-88. doi:10.2307/1797935

Russegger, J. (1841). Über die depression des todten Meers und des ganzen Jordan-Thals, vom See Tiberia bis zum Waddi el Chor. Annalen der Physik und Chemie, 53 (5), 179-194. 
Russegger, J. (1939). J. Rußegger in Palästina. Beilage zur Allgemeinen Zeitung, 381-382. 389.

Salmon, T. J., McCaw, C. M. G. (1936). The Level and Cartography of The Dead Sea. Palestine Exploration Quarterly, 68 (2), 103-111. doi: 10.1179/ peq.1936.68.2.103

Seetzen, U. J. (1810). A Brief Account of the Countries Adjoining the Lake of Tiberias, the Jordan and the Dead Sea. London, Palestine Association of London.

Seetzen, U. J. (1854). Reisen durch Syrien, Palästina, Phönicien, die Transjordan-länder, Arabia Petraea und Unter-Aegypten (4 voll.). Berlin, G. Reimer.

Seetzen, U. J. (2002). Unter Mönchen und Beduinen, Reisen in Palästina und Angrenzenden Ländern, 1805-1807, herausgegeben von A. Lichtenberger. Stuttgart-Vienne, Wissenschaftliche Buchgesellschaft.

Tristram, H. B. (1865). The land of Israel; a journal of travels in Palestine. London, Society for promoting christian knowledge.

Tristram, H. B. (1873). Land of Moab : travels and discoveries on the east side of the Dead Sea and the Jordan. London, John Murray.

Underhill, H. W. (1967). Dead Sea Levels and the P.E.F. Mark. Palestine Exploration Quarterly, 99 (1), 45-53. doi:10.1179/peq.1967.99.1.45

van de Velde, C. W. M. (1854). Narrative of a journey through Syria and Palestine in 1851 and 1852 (2 voll.). Edinburgh and London, Blackwood and s.

von Schubert, G. H. (1838). Reise in das Morgenland in den Jahren 1836 und 1837. 3 voll., Erlangen, Palm \& Enke.

Wildenbruch, C. V. (1850). Notes on the Physical Geography of Palestine. The Journal of the Royal Geographical Society of London, 20, 227-232. doi:10.2307/1798032

Wilson, C. (1900). The Dead Sea. Palestine Exploration Quarterly, 32 (4), 365-369. doi:10.1179/peq.1900.32.4.365

Wright, J. K. (1966). Human Nature in Geography. Fourteen Papers, 1925-1965. Cambridge, Harvard University Press. doi: 10.4159/harvard.9780674434639 